Research Articles: Behavioral/Cognitive

\title{
Correlates of auditory decision making in prefrontal, auditory, and basal lateral amygdala cortical areas
}

https://doi.org/10.1523/JNEUROSCI.2217-20.2020

Cite as: J. Neurosci 2020; 10.1523/JNEUROSCI.2217-20.2020

Received: 20 August 2020

Revised: 2 November 2020

Accepted: 26 November 2020

This Early Release article has been peer-reviewed and accepted, but has not been through the composition and copyediting processes. The final version may differ slightly in style or formatting and will contain links to any extended data.

Alerts: Sign up at www.jneurosci.org/alerts to receive customized email alerts when the fully formatted version of this article is published. 
Auditory Decision-Making

\title{
Correlates of auditory decision making in prefrontal, auditory, and basal lateral
} amygdala cortical areas

\author{
Julia L. Napoli ${ }^{*}$, Corrie R. Camalier ${ }^{*}+$, Anna Leigh Brown, Jessica Jacobs, Mortimer M. Mishkin and Bruno \\ B. Averbeckt \\ ${ }^{1}$ Laboratory of Neuropsychology, National Institute of Mental Health, National Institutes of Health, \\ Bethesda MD, 20892-4415
}

\section{tCorrespondence to:}

Corrie R. Camalier, Ph.D.

Laboratory of Neuropsychology, NIMH/NIH

Building 49 Room $1 \mathrm{~B} 80$

49 Convent Drive MSC 4415

Bethesda, MD 20892-4415

E-mail: corrie.camalier@duke.edu

Bruno B. Averbeck, Ph.D.

Laboratory of Neuropsychology, NIMH/NIH

Building 49 Room 1B80

49 Convent Drive MSC 4415

Bethesda, MD 20892-4415

E-mail: bruno.averbeck@nih.gov

*These authors contributed equally.

Acknowledgements: The authors would like to thank Dr. Richard Krauzlis (NEI/NIH), Dr. Barbara ShinnCunningham (Carnegie Mellon) and Dr. Brian Scott ( $\mathrm{NIMH} / \mathrm{NIH})$ for valuable input on task design and training, Dr. Richard Saunders (NIMH/NIH) for surgical assistance, and the NIH Section on Instrumentation for assisting in custom manufacture of recording chambers and grid. 
Auditory Decision-Making

Abstract

Spatial selective listening and auditory choice underlie important processes including attending to a speaker at a cocktail party and knowing how (or if) to respond. To examine task encoding and relative timing of potential neural substrates underlying these behaviors, we developed a spatial selective detection paradigm for monkeys, and recorded activity in primary auditory cortex (AC), dorsolateral prefrontal cortex (dIPFC) and the basolateral amygdala (BLA). A comparison of neural responses among these three areas showed that, as expected, $A C$ encoded the side of the cue and target characteristics before dIPFC and BLA. Interestingly, AC also encoded the monkey's choice before dIPFC and around the time of BLA. Generally, BLA showed weak responses to all task features except the choice. Decoding analyses suggested that errors followed from a failure to encode the target stimulus in both $\mathrm{AC}$ and dIPFC, but again, these differences arose earlier in AC. The similarities between $A C$ and dIPFC responses were abolished during passive sensory stimulation with identical trial conditions, suggesting that the robust sensory encoding in dIPFC is contextually gated. Thus, counter to a strictly PFC-driven decision process, in this spatial selective listening task, AC neural activity represents the sensory and decision information before dIPFC. Unlike in the visual domain, in this auditory task, the BLA does not appear to be robustly involved in selective spatial processing.

\section{Significance Statement}

We examined neural correlates of an auditory spatial selective listening task by recording single neuron activity in behaving monkeys from the amygdala, dorsal-lateral prefrontal cortex, and auditory cortex. We found that auditory cortex coded spatial cues and choice-related activity before dorsal-lateral prefrontal cortex or the amygdala. Auditory cortex also had robust delay period activity. Therefore, we found that auditory cortex could support the neural computations that underlie the behavioral processes in the task. 
Auditory Decision-Making

\section{Introduction}

Spatial selective listening is critical for solving everyday problems including the classic "cocktail party problem", which requires attending to one sound source amidst a noisy background of competing sources (Cherry, 1953). Common auditory spatial selective listening paradigms used in research with humans include modified Posner paradigms in which subjects detect auditory stimuli after being cued to a spatial location (Spence and Driver, 1994; Alho et al., 1999; McDonald and Ward, 1999; Mayer et al., 2007; Mayer et al., 2009; Roberts et al., 2009; Teshiba et al., 2013) and selective listening studies (Ahveninen et al., 2013; Frey et al., 2014; Bidet-Caulet et al., 2015). Previous work in humans has shown that auditory cortex (AC) plays an important role in spatial selective listening tasks, through interactions with prefrontal (Alho et al., 1999) and parietal (Deng et al., 2019) cortex. In addition to a role for these structures, previous studies in the visual domain in non-human primates have shown that the BLA contributes to spatial selective attention (Peck and Salzman, 2014; Costa et al., 2019).

There are only a few studies comparing multiple areas in auditory processes, especially in nonhuman primates, so we lack clear evidence on the relative contributions and timing of information between areas. Auditory processing is characterized by speed, especially relative to the visual system. In non-human primates, primary auditory cortex (AC) has response latencies of $\sim 20 \mathrm{~ms}$ (Camalier et al., 2012), compared to 40 ms for primary visual cortex (Schmolesky et al., 1998). AC is, however, further removed from the peripheral sensory receptors than primary visual cortex. This speed is consistent with a hypothesized role for the auditory system in rapid spatial alerting or orienting. However, the processing depth of primary AC has led some authors to suggest that it can also process cognitive factors such as choice, normally attributed to higher-order sensory areas (Naatanen et al., 2001; Nelken, 2004). Certainly, AC has been shown to reflect aspects of auditory decision making beyond sensory processing (Niwa et al., 2012; Tsunada et al., 2016; Christison-Lagay and Cohen, 2018; Huang et al., 2019), but it is unclear if this choice information is coming from PFC or another area (Lee et al., 2009; Plakke et al., 2015). A recent decision-making study in ferrets suggested that sensory information was encoded first in primary AC, but category information and the decision was encoded first in ferret dIFC, which is a premotor area potentially analogous to primate PFC (Yin et al., 2020). This would be consistent with auditory working memory data in non-human primates which suggests that a categorical "match" decision may emerge earlier in ventral PFC than AC (Bigelow et al., 2014). At present, the relative role of $A C$ and dIPFC, especially in spatial decision making, is unclear. Aside from the cortical sensory and prefrontal pathways, a BLA pathway for spatially selective processing and decision making is hypothesized to be fairly fast in the visual domain (Peck and Salzman, 2014; Costa et al., 2019), but whether the BLA is involved in auditory decision making in non-human primates is unknown.

To address these outstanding questions, here we describe an experiment in which we used a spatial selective detection paradigm for monkeys, grounded in spatially cued listening tasks used in the human studies discussed above. To investigate potential neural correlates of this task, we recorded single unit activity in primary AC (A1), dIPFC, and the BLA while the monkeys carried out the task. The dIPFC recordings were located in dorsal pre-arcuate cortex (primarily area 8A, see methods; Fig. 1B), which is the primary prefrontal target of the auditory "dorsal stream" arising from caudal belt and 
Auditory Decision-Making

parabelt, thought to be important for auditory spatial processing (Bon and Lucchetti, 1994; Hackett et al., 1999; Lanzilotto et al., 2013). Specifically these recordings targeted the zone between the principal sulcus and dorsal arcuate sulcus, at least $1 \mathrm{~mm}$ away from the arcuate sulcus, primarily corresponding to area 8Ad, but also potentially including the dorsal bank of $46 \mathrm{~d}$, caudal $8 \mathrm{Adv}$, and caudal border of $8 \mathrm{~b}$. Recordings in the amygdala targeted to the basal and lateral nuclei. Cortical auditory inputs to the amygdala caudal parabelt, terminate in the larger lateral nucleus (Yukie, 2002). However, the rostral superior temporal gyrus, which also indirectly receive auditory input, projects more broadly to the lateral and basal nuclei (Stefanacci and Amaral, 2002). We examined the strength and latency of signals at the single cell level related to the task across these areas. In AC and dIPFC a substantial fraction of neurons was selective to the location of the cue and the subsequent target. We found that AC preceded both IIPFC and BLA in sensory discrimination and also in the decision. Classification analyses of firing rate patterns in error trials indicated that errors during the task were usually the result of a failure to encode the first target stimulus in AC and also in dIPFC. A comparison of responses and timing with a control "passive listening" condition showed that sensory target related activity in dIPFC was almost completely abolished in the passive task, suggesting task-dependent gating of information to areas beyond sensory cortex.

\section{Methods}

The experiments were carried out using two adult male rhesus macaques (Macaca mulatta). The monkeys had access to food 24 hours a day and earned their liquid through task performance on testing days. Monkeys were socially pair housed. All procedures were reviewed and approved by the NIMH Animal Care and Use Committee.

\section{Experimental Setup}

The monkeys were operantly trained to perform a spatial selective listening paradigm. The task was controlled by custom software (Tucker Davis Technologies (TDT) System 3: OpenWorkbench and OpenDeveloper, TDT) which controlled multi-speaker sound delivery and acquired bar presses and eye movements. Eye movements were tracked using an Arrington Viewpoint eye tracking system (Arrington research) sampled at $1 \mathrm{kHz}$. Monkeys were seated in a primate chair facing a 19-Inch LCD monitor $40 \mathrm{~cm}$ from the monkey's eyes, on which the visual fixation spot was presented. Monkeys performed the task in a darkened, double-walled acoustically isolated sound booth (Industrial Acoustics Company, Bronx, NY). All auditory stimuli were presented from a speaker $10 \mathrm{~cm}$ from the left or right of the monkey's head. Juice rewards were delivered using a solenoid juice delivery system (Crist Instruments).

\section{Task Design and Stimuli}

The monkeys carried out a spatial selective listening task (Fig. 1), modeled after spatially cued tasks used in humans. The task required oculomotor fixation throughout the duration of the trial. Both spatial cues and target stimuli were auditory and the monkeys were required to respond when they detected a target embedded in masking noise presented on the cued side. Listening conditions (listen left/right) were blocked with two types of trials (match/foil) in each condition. At the start of each trial, the monkey was prompted to press a lever and fixate a central point on the screen. After a short delay (2.1-2.4 s), a $50 \mathrm{~ms} 4 \mathrm{kHz}$ square wave $(70 \mathrm{~dB})$ cue was played from a speaker on the left or right of the 
Auditory Decision-Making

midline. Frozen diotic white noise $(40 \mathrm{~dB})$ was then played from both the left and right speakers from $500 \mathrm{~ms}$ after the initial cue until the lever was released. Following a variable delay after noise onset (500, 800 or $1300 \mathrm{~ms}$ ) a $300 \mathrm{~ms} 1 \mathrm{KHz}$ square wave target sound was played from either the left or right speaker. If the target sound was on the same side as the cue, it was a match trial and the animal had to release the lever within 700 ms to receive a juice reward. If the target sound was on the opposite side as the cue, it was a foil trial and the monkey had to continue to hold the lever. Following a second interval of 800 or $1000 \mathrm{~ms}$ in foil trials, a second $1 \mathrm{KHz}$ match target was always played on the same side as the original cue. If the animal correctly released the lever following the second target in foil trials it was given a juice reward. Thus, both match and foil trials were identical in terms of reward expectation. If the choice was incorrect, there was a long "timeout" period before the next trial could be initiated. As in our previous work (Camalier et al., 2019) the use of square waves (which contain odd harmonics) allowed for wideband stimulation that was perceptually distinct, but whose broad spectral signature robustly activated large swaths of AC in a way that pure tones would not. Thus, similar to human paradigms, the stimuli could be kept identical across all sessions, independent of where recordings were carried out in AC, and data could be collapsed across sessions for analysis.

To achieve maximal effort and selective effects on neurons (as well as be able to analyze sources of errors), it was important that the targets be difficult to detect. Thus, several psychometric quality controls were included to ensure that the monkeys were consistently performing the task across sessions. The sound level of the target for the two monkeys was individually titrated to maintain performance at $\sim 70-80 \%$ correct (exact $71.14 \%$ ). Thus, the detection was difficult. The cue presentation was blocked to ensure the monkeys were able to maintain high accuracy on the task, as complex auditory tasks in monkey have traditionally been difficult to condition operantly (Scott and Mishkin, 2016; Rinne et al., 2017). Analysis of the first trial after the cue switched sides showed that animals were correct $76.13 \%$ of the time, indicating the monkeys were primarily using the cue in the task. For monkey 1, target tones were delivered at levels between 16-40 dB, with most tones in the 17-24 dB range. For monkey 2 target tones were delivered at levels between $27-45 \mathrm{~dB}$, with most tones in the 29$36 \mathrm{~dB}$ range. Within a session, the target sound varied $0-7 \mathrm{~dB}$ from trial to trial to ensure that the monkeys were responding to the target side and not to consistencies in (or guessing based on) sound level differences between speakers that may have resulted from otherwise undetectable differences in calibration between the two speakers. To further ensure accurate performance, periodic "catch trials" ( $10 \%$ with a $0-\mathrm{dB}$ target tone) were included to ensure that the monkeys were responding to the target and not timing their choices relative to the presentation of the cue or noise. To encourage motivation during foil trials (which were longer duration \& were thus more likely to be aborted), the "match/bar release" target after a foil sound was louder (and easier) than typical target sounds for each monkey (monkey 1: 27 or $30 \mathrm{~dB}$; monkey 2: 35 or $40 \mathrm{~dB}$ ).

Before the task was run, a battery of passive listening and mapping stimuli were played. Within this battery was a control condition of "passive listening". In this condition the monkey was presented with the task stimuli with trial types and timing matched to the selective listening task. However, the animals did not press or release a bar, fixate, or receive juice rewards. This task allowed us to compare sensory responses between active and passive task conditions. Monkeys were cued that this was a 
Auditory Decision-Making

passive condition as they did not have access to the lever or juice tube, and it was done as part of a passive-listening mapping battery, consistently before the start of the active task.

\section{Neurophysiological Recordings}

Monkeys were implanted with titanium headposts for head restraint before data collection began. Custom $45 \times 24 \mathrm{~mm}$ acrylic chambers were designed and fitted to the monkeys in a separate procedure. The chamber was aligned with the long axis oriented anterior-posterior. The placement allowed vertical grid access to the left dorsolateral prefrontal cortex (Fig. 1B; dorsal bank of the principal sulcus extending to dorsal arcuate but at least, $>1 \mathrm{~mm}$ away from arcuate sulcus, primarily corresponding to area $46 / 8 \mathrm{Ad}$, but also potentially including dorsal bank of $46 \mathrm{~d}$, caudal $8 \mathrm{Adv}$, and caudal border of $8 \mathrm{~b}$ ), the basal and lateral portions of the amygdala (entire dorsoventral extent), and auditory cortex (primarily A1 but including small portions of surrounding areas). A $1 \mathrm{~mm}$ grid was located inside the chamber for targeting (Fig. 1B, lower-right), and all penetrations were dorsal-ventral. This dorso-ventral trajectory was essential for targeting $A C$ tonotopic reversals. The chamber was custom fit to a 3D print of each monkey's skull generated by a CT scan before implantation. Recording areas were verified through a T1 scan of grid coverage with respect to underlying anatomical landmarks (Fig. 1B), combined with maps of frequency reversals and response latencies of single neurons to determine A1 location and extent (Camalier et al., 2012; Camalier et al., 2019). Recordings were mainly carried out in simultaneous AC and PFC sessions, with BLA sessions occurring later in the experiment, but some data included were from just one, or even three simultaneously recorded areas in a given session. We recorded the activity of 2,387 single neurons during the task ( $N=847(A C), N=968$ (dIPFC), and $N=572$ (BLA) across monkeys 1 $(\mathrm{N}=1540)$ and $2(\mathrm{~N}=847))$.

In both monkeys, we recorded using either 16 or 24 channel laminar "V-trodes" (Plexon, Inc, Dallas TX; 200-300 $\mu \mathrm{m}$ contact spacing, respectively). The electrodes allowed for identification of white matter tracts, further allowing identification of electrode location with respect to sulci and gyri. To ensure vtrodes went as straight as possible, sharpened guide tubes for the buried structures (AMY, AC) were advanced 10-15 mm above the structures. This was not possible for the PFC as it is a surface structure, but a guide tube was used to puncture overlying granulation tissue to permit a Vtrode to advance. Electrodes were advanced through the guide tubers to their target location (NAN microdrives, Nazareth, Israel) and allowed to settle for at least 1 hour before recording. Neural activity was recorded either primarily simultaneously ( $\mathrm{AC}$ and $\mathrm{PFC}$ ) or primarily individually (BLA), although there were some sessions in which all 3 areas were recorded from.

Multichannel spike and local field potential recordings were acquired with a 64-channel Tucker Davis Technology data acquisition system. Spike signals were amplified, filtered $(0.3-8 \mathrm{kHz})$, and digitized at $\sim 24.4 \mathrm{kHz}$. Spikes were initially sorted online on all-channels using real-time window discrimination. Digitized spike waveforms and timestamps of stimulus events were saved for sorting offline (Plexon sorter $V$ 3.3.5). Units were graded according to isolation quality (single or multiunit neurons). Single and multiunit recordings were analyzed separately, but patterns were similar, so they were combined. The acquisition software interfaced directly with the stimulus delivery system and both systems were controlled by custom software (OpenWorkbench and OpenDeveloper, controlling a RZ2, RX8, Tucker 
Auditory Decision-Making

Davis Technologies (TDT) System 3, Alachua, FL). For inclusion in analysis cells had to be present for at least 2 blocks and 80 trials over the session.

\section{Data analysis}

For the ANOVA and PSTH analysis, all trials on which monkeys released the lever in the correct interval were analyzed (71.14\% of all trials). Trials in which the monkey answered incorrectly (28.86\% of all trials), were excluded. The average number of correct trials analyzed for the ANOVA and PSTH analyses were 467.05 (AC: 480.11 , dIPFC: 471.72 , and BLA: 439.81 trials). We performed a $2 \times 2 \times 5$ ANOVA (cue $x$ target $x$ sound level) on the activity of single neurons. The choice is given by the interaction in this ANOVA. The dependent variable was the firing rates of individual neurons. Trials in which the monkeys correctly released the lever within $700 \mathrm{~ms}$ of the target and which were not catch trials (target $\mathrm{OdB}$ ), were analyzed. The firing rate of each cell was computed in $300 \mathrm{~ms}$ bins advanced in 25 ms increments. We separated the analysis into three different segments of time, locked to the time surrounding the individual presentations of the cue, noise, and first target.

Next, we created a population post-stimulus time histogram (PSTH) for the firing rates of the individual neurons with respect to cue condition (left/right) and trial condition (match/foil). For this analysis the firing rate of each cell was computed in $1 \mathrm{~ms}$ bins and smoothed with a 3 bin moving average. Data are plotted using 25 ms bins, but t-tests, to determine onset latencies, were computed on the $1 \mathrm{~ms}$ bins.

For the decoding analyses, we separately analyzed correct and error trials. A trial was considered correct if the monkey released the lever after the presentation of the appropriate target within 700 ms. All other trials were deemed incorrect. The average number of error trials analyzed for decoding was 123.53 (AC: 127.57 , dIPFC: 136.99, and BLA: 94.77). For neural analysis, the firing rate of each cell was computed in $100 \mathrm{~ms}$ bins and advanced in $25 \mathrm{~ms}$ increments. Decoding analyses were performed using leave-one-out cross-validation to predict which observations belong to each trial condition using the SVM classifier in Matlab. All decoding was done using pseudo-populations composed of all neurons recorded from a structure across all sessions. Trials were assigned randomly from the different sessions within each condition.

For the ANOVA analyses, we used 300 ms bins, as this provided additionally sensitivity to detect significant effects in neurons with low firing rates. We followed this up with the population analysis which used 1 ms bins, to optimize detection of onset latencies. Finally, we used 100 ms bins for the decoding analysis because the large number of neurons used in this analysis increases the signal-tonoise ratio for detecting effects, and therefore a smaller bin than was used for the ANOVA analysis allows us to detect timing effects more accurately.

For the decoding analyses, we calculated significant differences between correct and error trials using a bootstrap analysis (Efron and Tibshirani, 1998). We generated data according the null hypothesis that there were no differences between correct and error trials. We did this by sampling with replacement, from the combined set of correct and error trials, sets of bootstrap correct and error trials. Both the null correct and error bootstrap sets contained combinations of correct and error trials. 
Auditory Decision-Making

We then carried out the decoding analysis using the bootstrap trials to determine the decoding accuracy when correct and error trials were mixed. We did this 1000 times. We calculated the difference in fraction correct between correct and error trials in each time bin, for each set of bootstrap trials. This gave us 1000 differences sampled from the null distribution, between correct and error trials, in each time bin. We then compared the difference in the actual data to the differences in the null distribution, and computed a p-value, which was the relative rank of the true difference in the null distribution samples. That is to say, if the true difference was larger than, for example, 986 samples in the null distribution, it was significant with a two sided p-value of $2 x(1000-986) / 1000=0.028$.

\section{Results}

\section{Task and Behavior}

We recorded neural activity from 2 monkeys while they carried out a spatial selective listening task (Fig. 1A). At the start of each trial, the monkeys acquired central fixation (Fig. 1A), and pressed a bar. After a baseline hold period, an auditory stimulus (the cue) was presented from a speaker on the left or right of the monkey. After the cue there was a delay period during which white noise was played, continuing until bar release. Following the delay period, a second target stimulus was presented on the same (match) or opposite (foil) side as the cue, at different sound levels (Fig. 2). The monkeys were trained to release the bar if the cue and target stimulus were on the same side (match trials) and continue to hold if they were not on the same side (foil trials). In foil trials, following a second delay after the target stimulus, a third match target was played that was always on the same side. In match trials the mean response time was $374.9 \mathrm{~ms}$ (std = $27 \mathrm{~ms}$ ). Monkey 1 had a slightly faster mean response time of $358.3 \mathrm{~ms}$ (std $11.5 \mathrm{~ms}$ ) and Monkey 2 had a mean response time of $405.1 \mathrm{~ms}$ (std $19.9 \mathrm{~ms}$ ).

\section{Single cell encoding of task factors}

While the animals carried out the task, neural activity was recorded (Fig. 1B), from three areas: auditory cortex ( $A C, N=847)$, dorsal lateral prefrontal cortex (dIPFC, $N=968$ ) and the basal lateral amygdala ( $B L A, N=572$ ). We found neurons in all structures that responded to the presented cues (Fig. $3 \mathrm{~A}, 3 \mathrm{C}, 3 \mathrm{E}$ ) and the targets, or the interaction of cue and target (Fig. 3B, 3D, 3F). We assessed the encoding of each task factor in single neurons across the population by carrying out ANOVA analyses on correct trials, for each single neuron. With the ANOVA we examined the effects of cue location, target location, target sound level, and interactions (cue $x$ target codes decision), using spike counts in a 300 ms window, advanced by $25 \mathrm{~ms}$ (Fig. 4). During the cue period, we found that activity discriminated cues rapidly in AC (Fig. 4A). In dIPFC, activity discriminated cues as well, but the effect increased slowly (Fig 4E). The BLA, however, showed minimal cue discriminative activity, with the number of neurons coding cue location only slightly above chance (Fig. 4I). Note that cue location trials were blocked in the task, which led to small baseline, statistically significant, elevation of cue side encoding prior to cue presentation. Although the cue side was blocked, performance on the first trial after the cue switched sides was $76.13 \%$ and therefore the animals were attending to the cue. Although encoding peaked in $A C$ and dIPFC following the cue, elevated cue discrimination was maintained during the delay interval, 
Auditory Decision-Making

which was not affected by the white noise, in both AC and dIPFC. The BLA showed less delay period activity.

When the target stimulus was presented, it was rapidly and robustly encoded in AC (Fig. 4C). The dIPFC also encoded the target stimulus (Fig. 4G), although later than AC, which would be expected. The BLA only weakly encoded the target stimulus and only at about the time of the choice (Fig. 4K). The cue $x$ target interaction, which defined the choice in correct trials, was encoded first in AC (Fig. 4C), after which it was encoded in dIPFC (Fig. 4G). The cue $x$ target interaction, unlike the cue and target locations, was robustly encoded in the BLA (Fig. 4K). Sound level was also robustly encoded in AC (Fig. 4C) and less robustly in dIPFC (Fig. 4G) and BLA (Fig. 4K).

We also followed up this ANOVA with an additional ANOVA analysis that included both correct and error trials. This allowed us to dissociate the choice from the sensory processing reflecting the cue $x$ target interaction. When we carried out this analysis, we found that the choice was more robustly encoded than the cue $x$ target interaction across all areas (Fig. 4D, 4H, 4L) and most of the cue $x$ target interaction could be accounted for with the choice variable. Overall, all variables, including the delay period activity and the choice, were encoded first and most robustly by AC. The dIPFC did encode all task factors, but after AC. The BLA showed only weak encoding of the cue and the target but robustly encoded the choice.

In the next analysis, we compared encoding of the choice in fast and slow reaction time trials, to see if encoding of the choice (i.e. the interaction between cue side and target side in the ANOVA) differed (Fig 5). We performed a median split using the reaction times for all trials, both match and non-match, within a session. For non-match trials we used the release time after the second target as the RT. ANOVAs were run on each neuron twice, once on trials below the median reaction time, and once on trials above the median reaction time. We found, in all three areas, that the choice was encoded faster when the animals responded quickly than when the animals responded slowly. Only in auditory cortex did the activity related to the choice diverge before the average of the fast reaction times (Fig 5A). In both dIPFC and the BLA the activity diverged just before or after the average fast reaction time. Thus, the choice variable from the ANOVA depends on the timing of the motor response and is not completely determined by the timing of the auditory cues.

The results from the ANOVA analyses show the contribution of the neurons to each task factor. However, they do not illustrate whether single neurons code multiple task factors through time. Therefore, we also examined whether single neurons encoded more than one task factor, during each epoch (Fig. 6). It could be seen that many neurons coded more than one task factor and coded cue, for example, in both the cue and delay periods.

To further quantify whether neurons encoded more than one variable, we also estimated the fraction of neurons that encoded multiple factors using a single representative bin for each factor, centered on the time at which the population encoding of each factor peaked (Table 1). Most often, neurons that encoded the cue during the cue presentation continued to encode the cue during the delay interval. In AC, of the neurons that encoded the cue during cue presentation, $26.70 \%$ of them also 
Auditory Decision-Making

encoded the cue during the delay period. Neurons in dIPFC were most selective to encoding the cue during both the cue presentation and through the delay interval, with an overlap of $30.90 \%$. In the BLA, $25.00 \%$ of the neurons encoded the cue during both time periods. Neurons that encoded the cue also often eventually encoded the target, with an overlap of $17.05 \%, 10.11 \%$, and $8.33 \%$ in AC, dIPFC, and the BLA respectively. Most interestingly, while a relatively small portion of neurons encoded the cue through the delay period as well as eventually encoding the target in dIPFC and the BLA, AC did this with an overlap of $16.9 \%$. Neurons in $\mathrm{AC}$ were most likely to continue to encode other task variables, in comparison to the dIPFC and the BLA.

Next we examined finer time-scale encoding of several task factors. The ANOVA analysis used relatively large time windows to calculate sensitive statistics on potentially low firing rate neurons. These time windows, however, do not allow determination of precise onset times for task factors. To characterize onset times at a finer time scale, we calculated PSTHs using $1 \mathrm{~ms}$ time windows, smoothed with a 3-point moving average, for each neuron (Fig. 7 - plotted using 25 ms bins). We then carried out t-tests $(p<0.01$, uncorrected) in each bin to estimate the time at which the population in each area discriminated between conditions. We found that the cue was discriminated in AC at $25 \mathrm{~ms}$ (Fig. 7B) and in dIPFC at $65 \mathrm{~ms}$ (Fig. 7D) after stimulus onset. Using these small bins, the population of BLA neurons did not discriminate cue side, likely due to low firing rates (Fig. 7F). The target was discriminated in AC at $36 \mathrm{~ms}$ (Fig. 7G), in dIPFC at 169ms (Fig. 7I) and in the BLA at 185ms (Fig. 7K) after tone onset. Finally, the decision was discriminated in AC at $146 \mathrm{~ms}$ (Fig. 7H), in dIPFC at $321 \mathrm{~ms}$ (Fig. 7J) and in BLA at $266 \mathrm{~ms}$ (Fig. 7L) after target onset.

Next, we used a bootstrap analysis to determine whether onset latencies differed significantly between areas (Fig 7). We pulled samples of 100 neurons for each brain area and computed the time at which the two conditions diverged ( $p<0.05$, consecutive bins $>=6$ ) in each bootstrap sample. We did this 100 times to create a sample distribution. We then calculated a $95 \%$ confidence interval for the discrimination times for each area. If the confidence intervals overlapped, the brain areas were not deemed statistically significant. From this analysis, AC preceded both dIPFC and BLA in cue and target discrimination, and AC preceded dIPFC in decision discrimination. AC, however, did not statistically precede the BLA in decision discrimination.

\section{Decoding correct and error trials}

In the next analyses we used decoding to examine error trial activity. We were interested in which processes broke down in error trials. To examine this, we used leave-one-out cross validation on pseudo populations (see methods) to predict, using the neural activity, the side on which the cue was presented (Fig. 8), the side on which the target was presented (Fig. 9) and the choice (Fig. 10). The decoding model was first estimated using only correct trials. We then classified the error trials using the decoding model estimated on correct trials, to see if neural activity in error trials represented the stimuli that were presented, and the choice that was made. We found that in correct and error trials the neural population in both $A C$ and dIPFC rapidly predicted the cue location (Fig. 8A, D), and maintained prediction through the delay interval (Fig. 8B, D), consistent with the single-neuron results. The BLA did not discriminate clearly the cue side (Fig. 8G). There were no significant differences between correct 
Auditory Decision-Making

and error trials for cue encoding, and this finding was consistent through the delay interval. Therefore, the cue was correctly encoded in error trials.

When we decoded the target side using neural activity, we found that in correct trials the target location was robustly predicted by AC (Fig. 9C) and dIPFC (Fig. 9F). There was minimal prediction of the target in the BLA (Fig. 9l). In error trials, however, the target was not well predicted by any of the areas (Fig. 9). The correct and error trial predictions diverged ( $p<0.01$ bootstrap) 75 ms after target onset in $\mathrm{AC}$ and $125 \mathrm{~ms}$ after target onset in dIPFC.

In error trials, animals either released when they should not have, or did not release when they should have. When we predicted the choice, relative to what the monkeys should have done, we found an accurate prediction in correct trials in all 3 areas (Fig. 10C, 10F, 10I). Furthermore, in error trials, the predicted choice tended to fall below chance, which indicates that the neural activity is coding the choice the monkey made in error trials, as opposed to the choice the monkey should have made. However, this coding was only significantly below chance late in the choice period in AC (Fig. 10C). We used a smaller time bin of $5 \mathrm{~ms}$ in the rightmost column (Fig 10C,F,I) to more precisely determine the point at which the curves diverged. Consistent with the other analyses, we found that predictions in error and correct trials diverged statistically in auditory cortex (270 ms after target onset) and subsequently in dIPFC and BLA (275 and 300 ms after target onset).

Next, we examined the position of the population neural activity relative to the discrimination boundary, extracted from the decoding model. For the decoding analysis (Fig. 8-10), this quantity is thresholded in each trial and time-bin, and the time-bin in that trial is classified as either, e.g. cue left or cue right, depending on whether the position is positive or negative. However, the average distance to the decoding boundary provides a continuous estimate of how well the population discriminated the conditions vs. time (Fig. 11). In general, these analyses were consistent with the thresholded decoding analysis. Cue related activity diverged in correct and error trials, reflecting the cued side, and the activity in error trials matched the activity in correct trials (Fig. 11A, D, G). The breakdown in activity following target presentation could also be seen (Fig. 11B, E, H). However, there was some maintained coding of the target, particularly in auditory cortex (Fig. 11B), which may also be reflected in the decoding accuracy in error trials (Fig. 9C). Therefore, cue encoding is intact in error trials and target encoding is mostly but not completely absent. The choice encoding dynamics did reflect the fact that the wrong choice tended to be predicted by population activity (Fig. 11C, F, I). However, it could be seen that the activity diverged less than it did in correct trials, consistent with the lower decoding performance.

\section{Neural responses in the passive task}

In a final series of analyses, we analyzed data from a passive task, collected in each session before the main, active task data. The sensory stimulation in the passive task was identical to the stimulation in the active task, except the animals did not press a bar to initiate a trial, they did not release the bar to indicate their choice, and there was no juice tube so they could not be rewarded. When we examined encoding of cue location, we again found robust coding in AC (Fig. 12A). All of the 
Auditory Decision-Making

411

412

413

414

415

416

417

418

419

420

421

422

423

424

425

426

427

428

429

430

431

432

433

434

435

436

437

438

439

440

441

442

443

444

445

446

447

448

449 other signals, however, were much weaker. The cue responses in dIPFC dropped from a peak near 30\% in the active task to about $10 \%$ in the passive task (Fig. 12D). Interestingly, there was delay activity in the passive task, in AC (Fig. 12B), perhaps because the animals were highly over-trained. The delay activity in dIPFC was reduced from about $20 \%$ of the population to about $10 \%$ (Fig. 12E). There was also a small amount of target encoding in AC (Fig. 12C). Target encoding in dIPFC did not exceed chance (Fig. $12 \mathrm{~F})$. Encoding in the BLA only sporadically exceeded chance, perhaps due to type-I errors, or low-level encoding (Fig. 12G-I).

We also examined onset times, using small time-bins (Fig. 13). We found differences in responses in $\mathrm{AC}$ that depended on the side of the stimulus for the cue at $32 \mathrm{~ms}$ (Fig. 13B) and for the target at $53 \mathrm{~ms}$ (Fig. 13G). However, we did not detect population level differences in responses, using these small time bins, in dIPFC or BLA, which suggests that responses that reached significance in the ANOVA analyses were driven by low firing rates. Overall, beyond cue encoding in $\mathrm{AC}$, responses across all 3 areas were reduced in the passive task, relative to the active task.

Discussion

We trained monkeys on a selective listening task, based on tasks used in humans. The task required animals to detect a difficult to discriminate auditory stimulus, embedded in white noise. We found that AC encoded cues, targets, and decisions, prior to either dIPFC or the BLA. In addition, AC had delay activity that coded the location of the initial cue. It is not clear, however, whether the AC delay activity depended on dIPFC delay activity, or even parietal activity that we did not record. Activity in dIPFC closely followed activity in AC. The BLA, on the other hand, only minimally encoded cue and target activity. The BLA was strongly engaged, however, at the time of choice, although the choice related activity followed activity in AC. Therefore, the AC appears to support many of the functions required for auditory selective listening. This is in contrast to early visual areas, which represent visual features, but play a minimal role in decision making aspects of tasks (Britten et al., 1992; Zaksas and Pasternak, 2006).

Previous work has shown that AC neurons can encode non-sensory, choice-related activity (Niwa et al., 2012; Christison-Lagay and Cohen, 2018; Huang et al., 2019). The Huang et al. study found that whether a choice was predictable following a cue tone, based on the task condition, affected neural responses in $\mathrm{AC}$ to the tone. Therefore, $\mathrm{AC}$ encoded whether the response was determined by the first cue. Our results are consistent with this and other studies (Christison-Lagay and Cohen, 2018), in that we show that auditory cortex encodes the necessary response. However, in our task, the choice was not determined by the first cue, so choice related activity only followed the target. Our paradigm does not allow us to dissociate decision making from the motor response required to indicate the decision and therefore our choice coding could be related to either, though note that it begins well before monkeys man reaction of time of $\sim 400 \mathrm{~ms}$. We also show, that encoding in AC precedes encoding in dIPFC, and we dissociated through our fully crossed experimental design, encoding of cue location, target location, and the required response. Although it is possible that $A C$ inherits response encoding from a cortical area other than dIPFC, the anatomical organization of this system suggests it would have to be a nearby area, for example belt or parabelt auditory cortex (Romanski and Averbeck, 2009; Kajikawa et al., 2015; 
Auditory Decision-Making

Tsunada et al., 2016). Given that AC is deeper into the neural processing stream than, for example, primary visual cortex (Mizrahi et al., 2014), it is also possible that AC could have sufficiently sophisticated mechanisms to compute the required response locally. Though AC precedes PFC in the encoding of the decision in both correct and error trials, the responses across areas are also quite similar within this task ( $\sim 50$ ms differences). This tight temporal relationship between AC and dIPFC is contextually dependent. When responses during the passive condition were analyzed, the fraction of responsive neurons was reduced and responses were later in all areas relative to the task-related responses (and BLA was completely unresponsive, consistent with a primary role in reward guided behavior). Particularly, dIPFC showed a reduction of responses to the cue and delay activity and an abolishment of target related activity compared to the active task condition. This is consistent with data from the same animals and areas during a passive oddball task in which dIPFC activity was later ( 100 ms) and weaker than in AC (Camalier et al., 2019). Taken together it suggests that the strength and timing of the information transfer between AC and dIPFC can be flexibly allocated and is dependent on task demands. Lastly, comparison of the active and passive conditions highlights the sustained nonsensory motor/reward related activity in "primary" sensory cortex (AC)(Knyazeva et al., 2020).

Several of the analyses show that the neural responses recorded in this task were not straightforward sensory responses to the auditory stimuli. This was true across areas. For example, we found that the cue $x$ target interaction, which defines choices in correct trials, was less strongly encoded than the choices, when both correct and error trials were analyzed. In addition, when we split trials into those with fast and slow reaction times, we found that the neural representation of the decision was coded earlier when choices were made earlier. We also saw that much of the task related neural activity was reduced, although not eliminated, in the passive condition, when animals did not have to respond to the sensory cues.

Both prefrontal (Green et al., 2011; Bidet-Caulet et al., 2015) and parietal (Michalka et al., 2016; Deng et al., 2019; Deng et al., 2020) cortex have been shown to play important roles in auditory spatial attention in humans. AC has also been shown to have attention selective modulation of single neurons when targets and distractors are separated by frequency content (Atiani et al., 2009; Schwartz and David, 2018; O'Sullivan et al., 2019). Although we found clear responses related to the cued side in dIPFC, they followed AC. This was true of not only the sensory responses, but also the decision response. From our data it is not, however, possible to determine whether the delay period activity, which may represent sustained attention/working memory for the cue location, was sustained by AC, dIPFC, or their interaction. In addition, several of the spatial attention paradigms used in the human work required participants to attend or discriminate sounds in one location, while ignoring sounds on the contralateral side (Deng et al., 2019). It is possible that if we had required the monkeys to carry out complex perceptual discriminations at one location, while ignoring distractors at another location, we would have found stronger engagement of dIPFC. We did use a white masking noise, following the cue signal, to examine its effects on behavior and neural representations of the cue location. Although we did see some effects of the noise onset in the decoding analysis, effects which were stronger in AC than dIPFC, they were transient and resulted in increased decoding accuracy for the cued location. The increased accuracy may have followed from an overall increase in neural activity, which may have 
Auditory Decision-Making

improved decoding performance. Also, we did not record neural activity in parietal cortex, which may also play a role in the sustained delay period activity, although it would be interesting to consider inferior parietal cortex in future studies.

We found that the BLA played little role in encoding the cue location, and responses related to the choice followed responses in AC. This is inconsistent with previous reports of the BLA's involvement in visual-spatial attention (Peck et al., 2013). In these tasks, the amygdala neurons encoded the valence of stimuli, that were saccade targets, during delay periods (Peck and Salzman, 2014). There are several differences between these tasks, and ours, however. For example, the tasks used in Peck et al. were based on visual-spatial paradigms instead of an auditory-spatial paradigm, and they also required eye movements to spatial locations. Although the BLA receives auditory inputs(Yukie, 2002), these inputs may play a smaller role in the primate than they do in rodents (Munoz-Lopez et al., 2010). In rodents, auditory cues can be associated with shock in Pavlovian fear conditioning (Romanski and LeDoux, 1992). These studies have shown that the amygdala plays an important role in the associative process between cues and shocks. Although, the amygdala is also involved in reward guided behavior (Costa et al., 2016; Averbeck and Costa, 2017; Costa et al., 2019). We did find a small, although significant, population of amygdala neurons, that encoded the auditory cue and the auditory target. They did so, however, at long latencies. Therefore, the BLA appears to play a minimal role in the cognitive process of selective listening under reward-constant trials in highly trained animals. It is however possible that if we had primarily recorded from the lateral nucleus, which receives most of the direct auditory inputs (Yukie, 2002), we would have found more neurons related to aspects of our task.

The present study also shows a substantial dissociation of function between the BLA and dIPFC. This dissociation differs from the similarity between these structures seen in reinforcement learning (RL) tasks, in which both dIPFC and the BLA show substantial encoding of the identity of visual stimuli, the reward values associated with those stimuli, and reward outcomes (Bartolo et al., 2019; Costa et al., 2019). The primary difference between the BLA and dIPFC, in RL tasks, is that the dIPFC strongly encodes the direction of eye movements required to saccade to a rewarding visual stimulus (Bartolo et al., 2019), whereas the BLA encodes eye movement directions only at a low level (Costa et al., 2019). Thus, in RL tasks, the BLA and dIPFC show similar responses, which are also similar to those seen in the ventral striatum (Costa et al., 2019) and orbito-frontal cortex (Costa and Averbeck, 2020), with which the BLA is mono-synaptically connected. The current study, however, shows that in cognitive, auditory selective listening tasks, the BLA and dIPFC show different responses, until the animal makes a reward guided choice.

Conclusions

We found that AC encoded cues, targets, and decisions, before dIPFC, in an auditory selective listening task. We also found that AC had delay period activity. The BLA had minimal cue or target activity, although it did encode decision activity. The decision related activity in the BLA, however, followed decision related activity in AC. Overall, this suggests that AC may carry out most important 
Auditory Decision-Making

528

529

530

531

532

533

534

535

536

537

computations relevant to auditory selective listening. The main caveat is that it is not possible to determine whether delay period activity, which likely critically underlies performance in this task, is supported by AC in the absence of dIPFC or parietal cortex. Future work, for example inactivating dIPFC and/or parietal cortex (Plakke et al., 2015), while recording in AC, could clarify this question. 
Auditory Decision-Making

\section{References}

Ahveninen J, Huang S, Nummenmaa A, Belliveau JW, Hung AY, Jaaskelainen IP, Rauschecker JP, Rossi S, Tiitinen H, Raij T (2013) Evidence for distinct human auditory cortex regions for sound location versus identity processing. Nat Commun 4:2585.

Alho K, Medvedev SV, Pakhomov SV, Roudas MS, Tervaniemi M, Reinikainen K, Zeffiro T, Naatanen R (1999) Selective tuning of the left and right auditory cortices during spatially directed attention. Brain Res Cogn Brain Res 7:335-341.

Atiani S, Elhilali M, David SV, Fritz JB, Shamma SA (2009) Task difficulty and performance induce diverse adaptive patterns in gain and shape of primary auditory cortical receptive fields. Neuron 61:467-480.

Averbeck BB, Costa VD (2017) Motivational neural circuits underlying reinforcement learning. Nature Neuroscience 20:505-512.

Bartolo R, Saunders RC, Mitz A, Averbeck BB (2019) Dimensionality, information and learning in prefrontal cortex. bioRxiv:823377.

Bidet-Caulet A, Buchanan KG, Viswanath H, Black J, Scabini D, Bonnet-Brilhault F, Knight RT (2015) Impaired Facilitatory Mechanisms of Auditory Attention After Damage of the Lateral Prefrontal Cortex. Cereb Cortex 25:4126-4134.

Bigelow J, Rossi B, Poremba A (2014) Neural correlates of short-term memory in primate auditory cortex. Front Neurosci 8:250.

Bon L, Lucchetti C (1994) Ear and eye representation in the frontal cortex, area 8b, of the macaque monkey: an electrophysiological study. Exp Brain Res 102:259-271.

Britten KH, Shadlen MN, Newsome WT, Movshon JA (1992) The analysis of visual motion: a comparison of neuronal and psychophysical performance. J Neurosci 12:4745-4765.

Camalier CR, Scarim K, Mishkin M, Averbeck BB (2019) A Comparison of Auditory Oddball Responses in Dorsolateral Prefrontal Cortex, Basolateral Amygdala, and Auditory Cortex of Macaque. J Cogn Neurosci:1-11.

Camalier CR, D'Angelo WR, Sterbing-D'Angelo SJ, de la Mothe LA, Hackett TA (2012) Neural latencies across auditory cortex of macaque support a dorsal stream supramodal timing advantage in primates. Proc Natl Acad Sci U S A 109:18168-18173.

Cherry EC (1953) Some Experiments on the Recognition of Speech, with One and with 2 Ears. J Acoust Soc Am 25:975-979.

Christison-Lagay KL, Cohen YE (2018) The Contribution of Primary Auditory Cortex to Auditory Categorization in Behaving Monkeys. Front Neurosci 12:601.

Costa VD, Averbeck BB (2020) Primate orbitofrontal cortex codes information relevant for managing explore-exploit tradeoffs. J Neurosci.

Costa VD, Mitz AR, Averbeck BB (2019) Subcortical Substrates of Explore-Exploit Decisions in Primates. Neuron 103:533-545 e535.

Costa VD, Dal Monte O, Lucas DR, Murray EA, Averbeck BB (2016) Amygdala and Ventral Striatum Make Distinct Contributions to Reinforcement Learning. Neuron 92:505-517.

Deng Y, Choi I, Shinn-Cunningham B (2020) Topographic specificity of alpha power during auditory spatial attention. Neuroimage 207:116360.

Deng Y, Reinhart RM, Choi I, Shinn-Cunningham BG (2019) Causal links between parietal alpha activity and spatial auditory attention. Elife 8. 
Auditory Decision-Making

Efron B, Tibshirani RJ (1998) An introduction to the bootstrap. New York: Chapman \& Hall.

Frey JN, Mainy N, Lachaux JP, Muller N, Bertrand O, Weisz N (2014) Selective modulation of auditory cortical alpha activity in an audiovisual spatial attention task. J Neurosci 34:6634-6639.

Green JJ, Doesburg SM, Ward LM, McDonald JJ (2011) Electrical neuroimaging of voluntary audiospatial attention: evidence for a supramodal attention control network. J Neurosci 31:3560-3564.

Hackett TA, Stepniewska I, Kaas JH (1999) Prefrontal connections of the parabelt auditory cortex in macaque monkeys. Brain Res 817:45-58.

Huang Y, Heil P, Brosch M (2019) Associations between sounds and actions in early auditory cortex of nonhuman primates. Elife 8.

Kajikawa Y, Frey S, Ross D, Falchier A, Hackett TA, Schroeder CE (2015) Auditory properties in the parabelt regions of the superior temporal gyrus in the awake macaque monkey: an initial survey. J Neurosci 35:4140-4150.

Knyazeva S, Selezneva E, Gorkin A, Ohl FW, Brosch M (2020) Representation of Auditory Task Components and of Their Relationships in Primate Auditory Cortex. Frontiers in Neuroscience 14.

Lanzilotto M, Perciavalle V, Lucchetti C (2013) Auditory and visual systems organization in Brodmann Area 8 for gaze-shift control: where we do not see, we can hear. Front Behav Neurosci 7:198.

Lee JH, Russ BE, Orr LE, Cohen YE (2009) Prefrontal activity predicts monkeys' decisions during an auditory category task. Front Integr Neurosci 3:16.

Mayer AR, Harrington DL, Stephen J, Adair JC, Lee RR (2007) An event-related fMRI Study of exogenous facilitation and inhibition of return in the auditory modality. J Cogn Neurosci 19:455-467.

Mayer AR, Mannell MV, Ling J, Elgie R, Gasparovic C, Phillips JP, Doezema D, Yeo RA (2009) Auditory orienting and inhibition of return in mild traumatic brain injury: a FMRI study. Hum Brain Mapp 30:4152-4166.

McDonald JJ, Ward LM (1999) Spatial relevance determines facilitatory and inhibitory effects of auditory covert spatial orienting. J Exp Psychol Human 25:1234-1252.

Michalka SW, Rosen ML, Kong L, Shinn-Cunningham BG, Somers DC (2016) Auditory Spatial Coding Flexibly Recruits Anterior, but Not Posterior, Visuotopic Parietal Cortex. Cereb Cortex 26:1302-1308.

Mizrahi A, Shalev A, Nelken I (2014) Single neuron and population coding of natural sounds in auditory cortex. Curr Opin Neurobiol 24:103-110.

Munoz-Lopez MM, Mohedano-Moriano A, Insausti R (2010) Anatomical pathways for auditory memory in primates. Front Neuroanat 4:129.

Naatanen R, Tervaniemi M, Sussman E, Paavilainen P, Winkler I (2001) "Primitive intelligence" in the auditory cortex. Trends Neurosci 24:283-288.

Nelken I (2004) Processing of complex stimuli and natural scenes in the auditory cortex. Curr Opin Neurobiol 14:474-480.

Niwa M, Johnson JS, O'Connor KN, Sutter ML (2012) Activity related to perceptual judgment and action in primary auditory cortex. J Neurosci 32:3193-3210. 
Auditory Decision-Making

O'Sullivan J, Herrero J, Smith E, Schevon C, McKhann GM, Sheth SA, Mehta AD, Mesgarani N (2019) Hierarchical Encoding of Attended Auditory Objects in Multi-talker Speech Perception. Neuron 104:1195-1209 e1193.

Peck CJ, Salzman CD (2014) Amygdala neural activity reflects spatial attention towards stimuli promising reward or threatening punishment. eLife 3.

Peck CJ, Lau B, Salzman CD (2013) The primate amygdala combines information about space and value. Nature Neuroscience 16:340-348.

Plakke B, Hwang J, Romanski LM (2015) Inactivation of Primate Prefrontal Cortex Impairs Auditory and Audiovisual Working Memory. J Neurosci 35:9666-9675.

Rinne T, Muers RS, Salo E, Slater H, Petkov Cl (2017) Functional Imaging of Audio-Visual Selective Attention in Monkeys and Humans: How do Lapses in Monkey Performance Affect Cross-Species Correspondences? Cereb Cortex 27:3471-3484.

Roberts KL, Summerfield AQ, Hall DA (2009) Covert auditory spatial orienting: an evaluation of the spatial relevance hypothesis. J Exp Psychol Hum Percept Perform 35:1178-1191.

Romanski LM, LeDoux JE (1992) Equipotentiality of thalamo-amygdala and thalamo-corticoamygdala circuits in auditory fear conditioning. J Neurosci 12:4501-4509.

Romanski LM, Averbeck BB (2009) The primate cortical auditory system and neural representation of conspecific vocalizations. Annu Rev Neurosci 32:315-346.

Schmolesky MT, Wang Y, Hanes DP, Thompson KG, Leutgeb S, Schall JD, Leventhal AG (1998) Signal timing across the macaque visual system. J Neurophysiol 79:3272-3278.

Schwartz ZP, David SV (2018) Focal Suppression of Distractor Sounds by Selective Attention in Auditory Cortex. Cereb Cortex 28:323-339.

Scott BH, Mishkin M (2016) Auditory short-term memory in the primate auditory cortex. Brain Res 1640:264-277.

Spence CJ, Driver J (1994) Covert Spatial Orienting in Audition - Exogenous and Endogenous Mechanisms. J Exp Psychol Human 20:555-574.

Stefanacci L, Amaral DG (2002) Some observations on cortical inputs to the macaque monkey amygdala: an anterograde tracing study. J Comp Neurol 451:301-323.

Teshiba TM, Ling J, Ruhl DA, Bedrick BS, Pena A, Mayer AR (2013) Evoked and Intrinsic Asymmetries during Auditory Attention: Implications for the Contralateral and Neglect Models of Functioning. Cerebral Cortex 23:560-569.

Tsunada J, Liu AS, Gold JI, Cohen YE (2016) Causal contribution of primate auditory cortex to auditory perceptual decision-making. Nat Neurosci 19:135-142.

Yin P, Strait DL, Radtke-Schuller S, Fritz JB, Shamma SA (2020) Dynamics and Hierarchical Encoding of Non-compact Acoustic Categories in Auditory and Frontal Cortex. Curr Biol 30:1649-1663 e1645.

Yukie M (2002) Connections between the amygdala and auditory cortical areas in the macaque monkey. Neurosci Res 42:219-229.

Zaksas D, Pasternak T (2006) Directional signals in the prefrontal cortex and in area MT during a working memory for visual motion task. J Neurosci 26:11726-11742. 
Auditory Decision-Making

667

668

669

670

Figure 1. Task design and recording locations. (A) Structure of the spatial selective listening task. Cue conditions (listen left/right) were blocked with two types of trials (match/foil) in each condition. To begin each trial, the animal must depress a lever and maintain fixation at a central point on the screen. After a short delay (2.1-2.4 s), the animal heard a $4 \mathrm{kHz}$ square wave cue from a speaker on the left or right of its head. A continuous white noise was played $500 \mathrm{~ms}$ after the initial cue to make target detection difficult. In match trials, the animal heard a $1 \mathrm{kHz}$ match target (various levels, see Methods) after some stimulus onset asynchrony (SOA) $(500,800$ or $1300 \mathrm{~ms})$ on the same side as the cue. If the animal released the lever within $700 \mathrm{~ms}$ of the match target, a fixed juice reward was delivered. In foil trials, the animal heard a $1 \mathrm{kHz}$ foil target on the opposite side as the cue after the same SOA. The animal had to continue to hold down the lever until a $1 \mathrm{kHz}$ match target was presented (after 800 or $1000 \mathrm{~ms}$ ) on the same side as the cue. If the animal released the bar within $700 \mathrm{~ms}$ of the match target, a fixed juice reward was delivered. The "passive listening" control condition was identical to the active task except the monkey listened passively and did not press a lever, fixate, respond, or receive juice. (B) Recording locations of single neurons across auditory cortex (AC), dorsolateral prefrontal cortex (dIPFC) and the basal lateral amygdala (BLA). (Top) Patch of dIPFC recording area morphed to anatomical landmarks. (Middle) AC grid coverage on region A1 of auditory cortex based on topography, latency and frequency reversals. (Bottom) Region of interest highlighted in blue--the entire left basolateral amygdala--targeted by v-trodes. In all three areas, we selectively recorded from the left hemisphere of the animal. The lower right image shows a structural MRI with contrast agent (betadine gel) in chamber grid holes for targeting. Recording locations and trajectories were further verified using tungsten electrodes inserted through grid locations to target areas. Yellow lines in each image show an approximate trajectory.

Figure 2. Auditory target sound level and accuracy. Trial performance compared against the target sound level in decibels. Numbers below line indicate percentage of trials across sessions at that sound level. Note, catch trials $(0 \mathrm{~dB})$ are not plotted, so percentages do not add to 1 . Bars at each point represent the standard error. Mean values were first calculated for each session, and then means were taken across sessions in which the indicated sound level was used. The standard error of the mean was calculated across sessions where the number of sessions are: Monkey 1: $16-20 \mathrm{~dB}(\mathrm{~N}=53) ; 21-25 \mathrm{~dB}(\mathrm{~N}$ = 19); $26-30 \mathrm{~dB}(\mathrm{~N}=53), 36-40 \mathrm{~dB}(\mathrm{~N}=1$ - data not shown); Monkey 2: $26-30 \mathrm{~dB}(\mathrm{~N}=42) ; 31-35 \mathrm{~dB}(\mathrm{~N}=$ 42); $36-40 \mathrm{~dB}(\mathrm{~N}=42) ; 41-45 \mathrm{~dB}(\mathrm{~N}=9)$.

Figure 3. Example neurons. Left hand panel shows rasters of single trials, right hand panel show $p$-value from ANOVA, for the indicated factor. Only correct trials are shown. X-axis for $p$-value plots shows right hand edge of $300 \mathrm{~ms}$ bin used for ANOVA. A. Example neuron from cue epoch in AC. B. Example AC neuron showing responses to target. Plotted $p$-values are for target factor. C. Example dIPFC neuron showing responses to cue. D. Example dIPFC neuron showing responses to target. Plotted $p$-values are for cue $x$ target interaction. E. Example BLA neuron showing responses to cue. F. Example BLA neuron showing response to cue $x$ target interaction.

Figure 4. ANOVA analysis. Recording of single neurons from caudal AC (A1, lateral belt), dIPFC and BLA while monkeys are performing a spatial selective listening task. A 2 × 2 factor ANOVA (Cue side $\times$ Target side, $p<0.05$ ) using $300 \mathrm{~ms}$ bins sliding at $25 \mathrm{~ms}$. Bin endpoint was used to align time on the $x$-axis, i.e. $300 \mathrm{~ms}$ is a bin from 0 to $300 \mathrm{~ms}$. Bars above each plot represent the bins in which a statistically significant fraction of neurons encode each factor by color $(p<0.01$; binomial test). (A, E, I) During presentation of the cue, neurons respond differentially to the cue location. (B, F, J) Post-cue, a substantial fraction of neurons is selective to cue side, during the delay period, in both AC and dIPFC. (C, G, K) Post-target presentation, a substantial portion of neurons in all three areas of interest are selective 
Auditory Decision-Making

to the choice. (D, H, L) Post-target presentation analysis including error trials shows choice encoding over and above cue $x$ target interaction. Thus, choices are not a direct reflection of sensory input.

Figure 5. Median reaction time split ANOVA analysis. Recording of single neurons from AC, dIPFC and BLA while monkeys were performing the task. The results are from a 2 × 2 ANOVA (Cue side $x$ Target side, $p<0.05$ ) using $300 \mathrm{~ms}$ bins sliding at $25 \mathrm{~ms}$. Only the interaction term (Response) is plotted on the graph. Bin endpoint was used to align time on the $x$-axis. The trials for all neurons were split into fast and slow reaction time by the median reaction time within a session and separate ANOVAs were run for each set of trials. Paired t-tests $(p<0.01$, consecutive bins $>=3$ ) were computed to determine significance between fraction of significant neurons assessed in each reaction time split. Bars above each plot represent the bins in which a statistically significant difference was seen between response to trials with fast reaction times vs. slow reaction times.

Figure 6. Contribution of individual neuron selectivity to the populational representation. $2 \times 2$ ANOVA ( $p<0.05$ ) using $300 \mathrm{~ms}$ bins sliding at $25 \mathrm{~ms}$ conducted on each individual neuron. Neurons are plotted along the $y$-axis and the time is on the $x$-axis. Bin endpoint was used to align time on the $x$-axis. Grey bars represent the times in which a neuron was significant for that task factor. Blue column displays encoding of the cue during the cue presentation, grey column shows the cue encoding during the delay period, red column shows the target encoding during target presentation and yellow column shows the response encoding during the target period.

Figure 7. Post-stimulus time histograms (PSTHs). Mean normalized firing rates of neurons plotted using non-overlapping $25 \mathrm{~ms}$ bins smoothed with a 3-point moving average. Only neurons significant for the corresponding factor (i.e. cue, target or cue $x$ target) were included in this analysis. Bin midpoint was used to align time on the $x$-axis. Analysis was conducted to assess precise timing of changes in neuronal firing rates in AC, dIPFC and BLA. Paired t-tests were performed on all bins to determine significant difference in firing rates. Bootstrapping analysis was performed to directly compare timing differences in different brain areas. Vertical bars with stars indicate non-overlapping $95 \%$ confidence intervals for discrimination times between areas. $(A, C, E)$ compares conditions that are identical in cue side but vary in target side, as a measure of sensory identification. (B, D, F) compares conditions that are identical in target side but vary in the cue location (left or right). (G, I, K) Conditions are matched for cue side but vary in target side. $(\mathrm{H}, \mathrm{J}, \mathrm{L})$ Conditions shown have opposite cue sides but matched target side.

Figure 8. Classification analysis to cue location factor comparing correct and error trials. Analysis was performed using $100 \mathrm{~ms}$ bins, sliding at $25 \mathrm{~ms}$. Bin endpoint was used to align time on the x-axis. Analysis performed using leave-one-out cross-validation to predict which observations belong to each cue condition. Bootstrap test performed with 1000 pseudorandom samples. No significant difference in classification rates was found between correct and error trials in any brain region during any time bin.

Figure 9. Classification analysis to target factor by correct or error. Analysis was performed using $100 \mathrm{~ms}$ bins, sliding at $25 \mathrm{~ms}$. Bin endpoint was used to align time on the $\mathrm{x}$-axis. Grey shaded areas represent timepoints where correct and error classification rates differ ( $p<0.01$, bootstrap). (C) In AC, a significant difference is seen in classification rates during the target epoch that begins after $75 \mathrm{~ms}$ and ends after $400 \mathrm{~ms}$. (F) In dIPFC, the difference in classification rates begins slightly later and ends slightly earlier, starting at $125 \mathrm{~ms}$ post-target and ending at $375 \mathrm{~ms}$ post-target.

Figure 10. Classification analysis to choice. Grey bar indicates timepoints where correct and error classification rates differ, red bar indicates timepoints where error trials were significantly below chance 
Auditory Decision-Making

(0.5). Bin endpoint was used to align time on the $x$-axis. The reaction time for detect trials is shown as a dotted line, with the standard deviation shaded, in panels C, F and I. (C) Differences between correct and error trials in AC were from (270ms to 500ms), (F) In dIPFC from ( $275 \mathrm{~ms}$ to $500 \mathrm{~ms}$ ) and (I) In BLA from (300ms to $500 \mathrm{~ms})$.

Figure 11. Distance to classification boundary derived from support vector machine classifier. Analyses were conducted using $200 \mathrm{~ms}$ bins sliding at $25 \mathrm{~ms}$. Bin endpoint was used to align to the $\mathrm{x}$-axis. Error trials were defined as trials in which the animal responded incorrectly or responded outside of the allotted reaction time window. Correct trials are presented in shades of blue and error trials in shades of red. The reaction time for detect trials is shown as a dotted line, with the standard deviation shaded, in panels $C, F$ and I. (A, D, G) Conditions were separated by whether a trial was cued on the left or right side of the animal. $(B, E, H)$ Conditions were separated by whether the first target was presented on the left or right side of the animal. $(C, F, I)$ Conditions were separated by whether there was a response or no response made by the animal, i.e. if it was a detect or foil trial, respectively. For error trials, conditions were separated by whether there should have been a response or no response, regardless of what the animal chose to do.

Figure 12. Passive task data. ANOVA analysis of data from passive condition, which was identical to the task, except the monkey was simply required to sit passively and listen to the task structure's sounds. Bars above each plot represent the bins in which a statistically significant fraction of neurons encode each factor by color $(p<0.01)$. ( $A, D, G)$ During presentation of the cue, neurons respond differentially to the cue location. $(B, E, H)$ Post-cue, neurons are selective to cue side, during the delay period, in both $A C$ and dIPFC, but weakly, compared to the active task. (C, F, I) Post-target presentation, only AC encodes the target, and none of the areas encode the response.

Figure 13. Passive data. Post-stimulus time histograms (PSTHs). Mean normalized firing rates of neurons plotted using non-overlapping $25 \mathrm{~ms}$ bins smoothed with a 3 -point moving average. Bin midpoint was used to align time on the $x$-axis. Analysis was conducted to assess precise timing of changes in neuronal firing rates in AC, dIPFC and BLA. Paired t-tests were performed on all bins to determine significant difference in firing rates. $(A, C, E)$ compares conditions that are identical in cue side but vary in target side, as a measure of sensory identification. ( $B, D, F)$ compares conditions that are identical in target side but vary in the cue location (left or right). (G, I, K) Conditions are matched for cue side but vary in target side. $(\mathrm{H}, \mathrm{J}, \mathrm{L})$ Conditions shown have opposite cue sides but matched target side. 
A

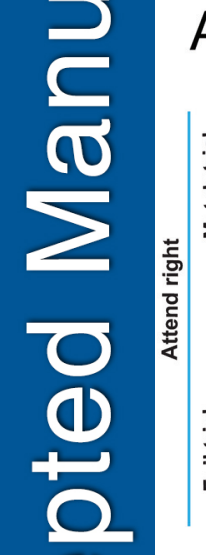

(1)

3

3

O

○

5

(b)
Lever press Cue Noise
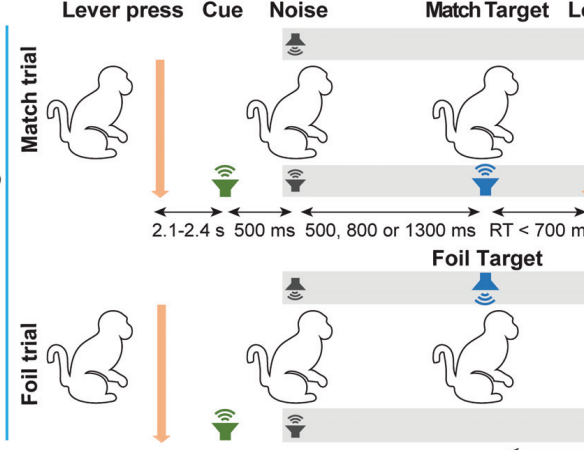

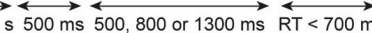

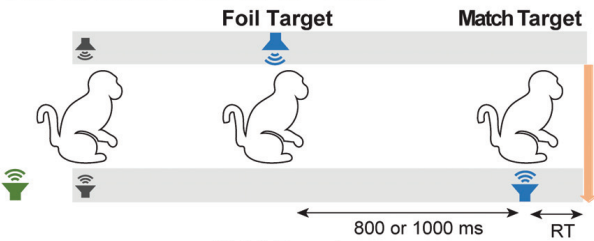

要

$\Xi$
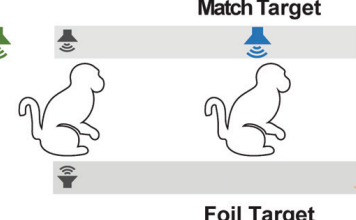

s

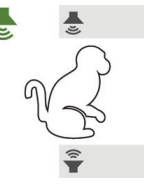

Match Target
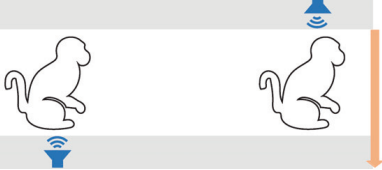

(1) Cue: $50 \mathrm{~ms} 4 \mathrm{kHz}$

(i) Noise: white noise

Target: $300 \mathrm{~ms}$
$1 \mathrm{kHz}$ wave

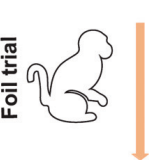

B
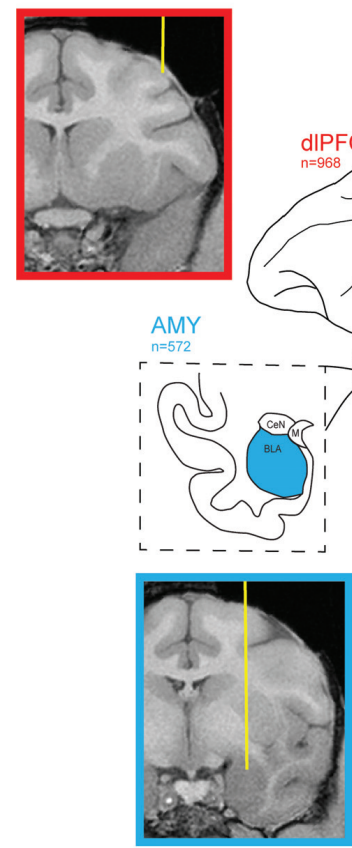
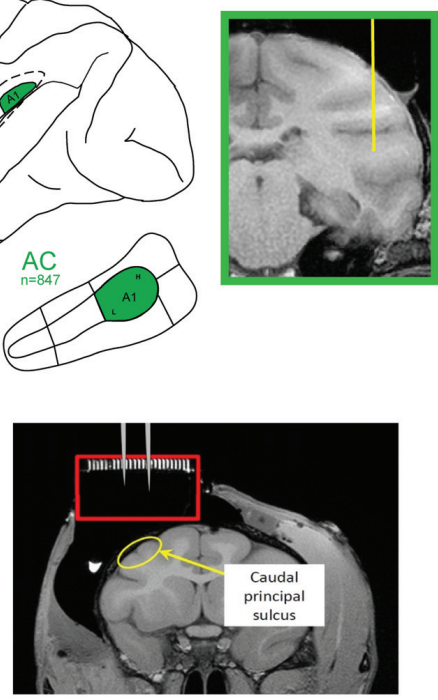

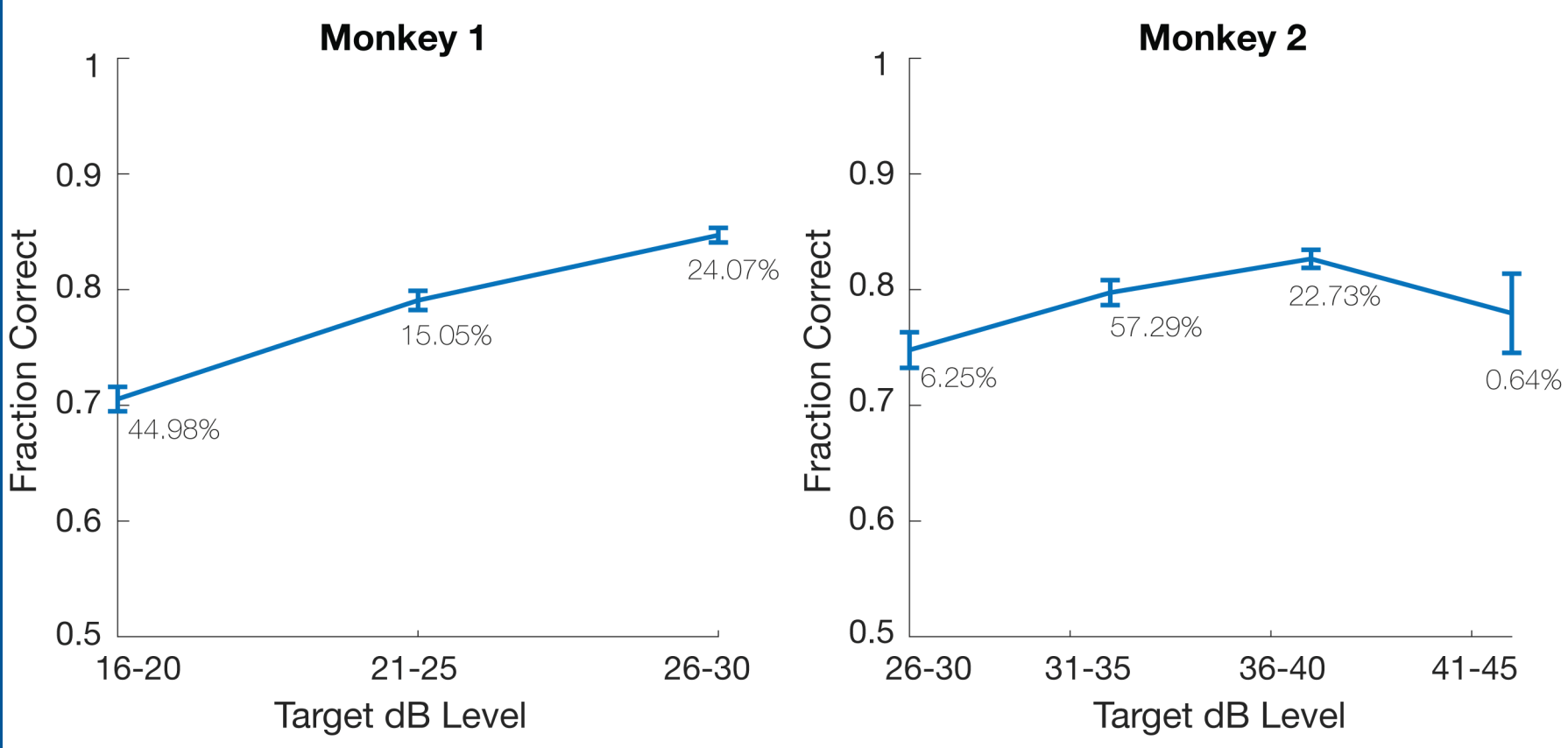
${ }^{A}{ }_{A C}$

画

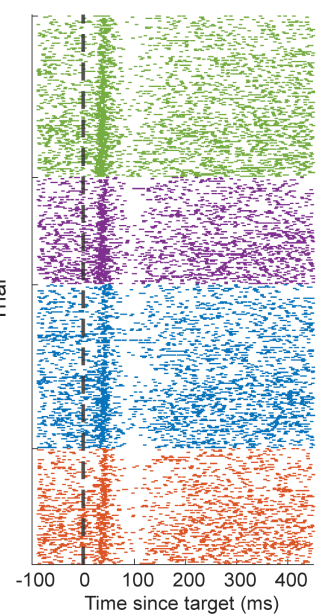

C

\section{dIPFC}
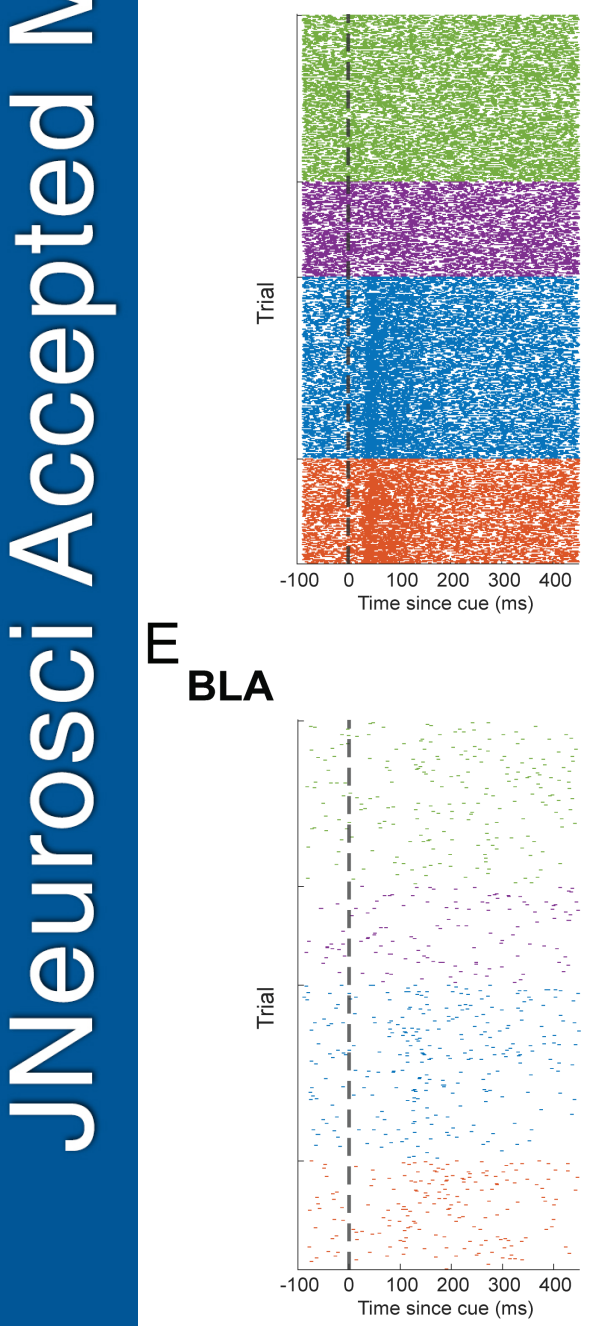

\section{Cue Epoch}
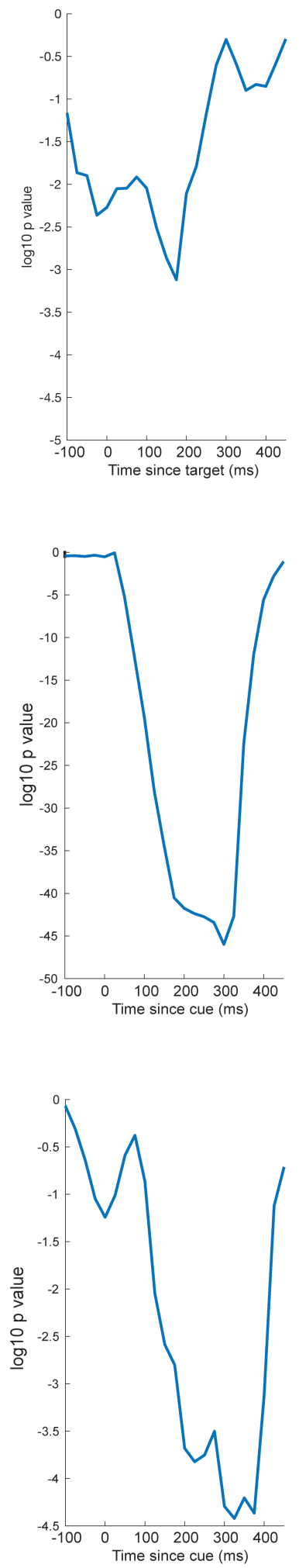

B

Target Epoch
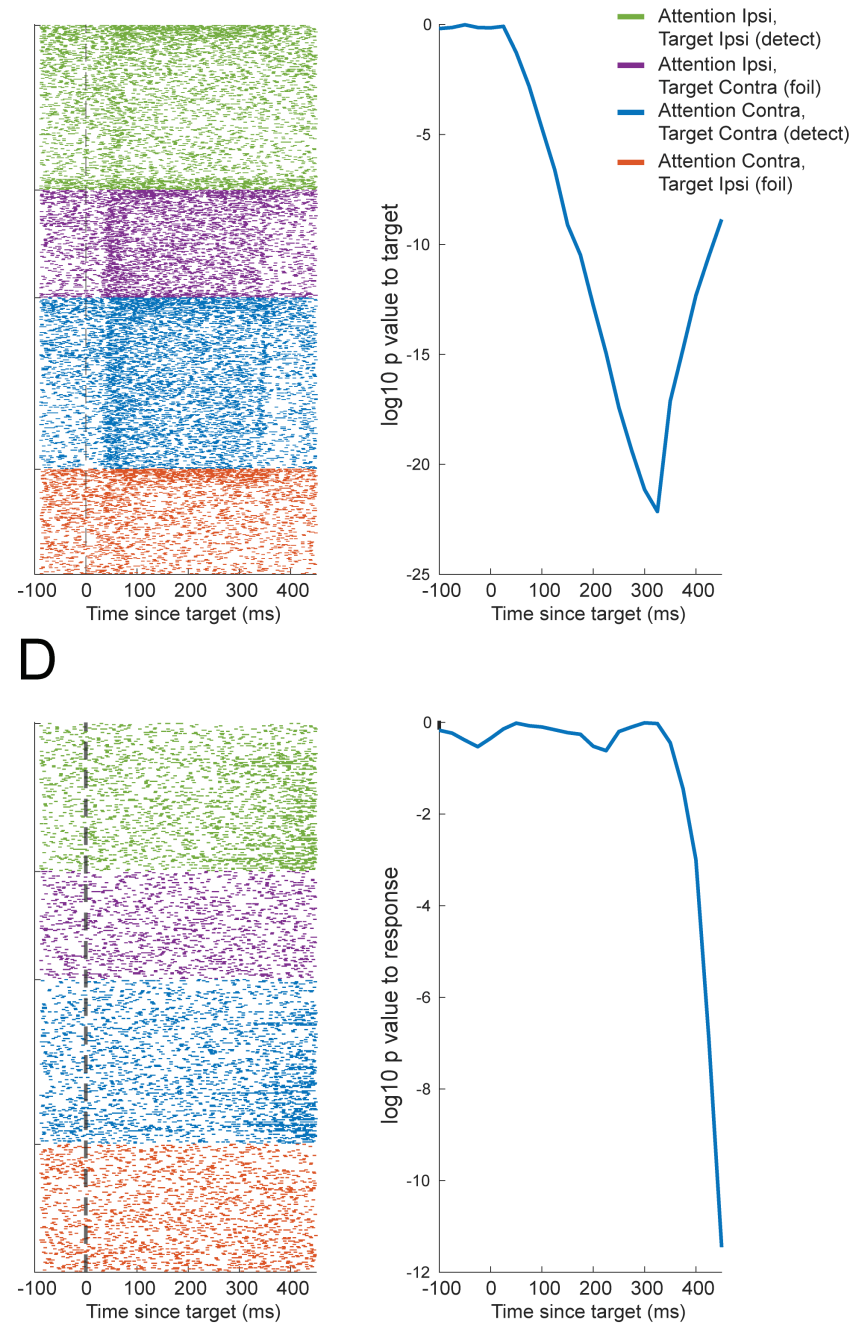

F
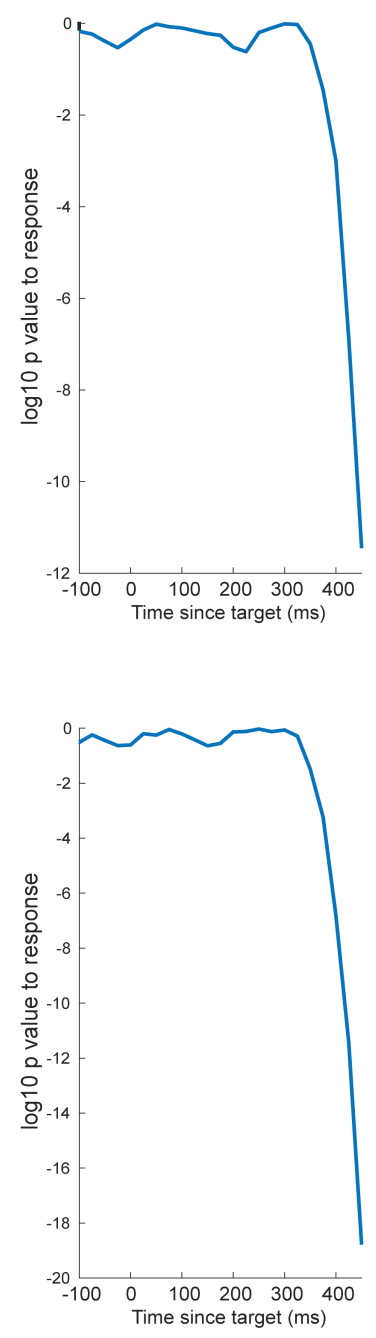
A

AC

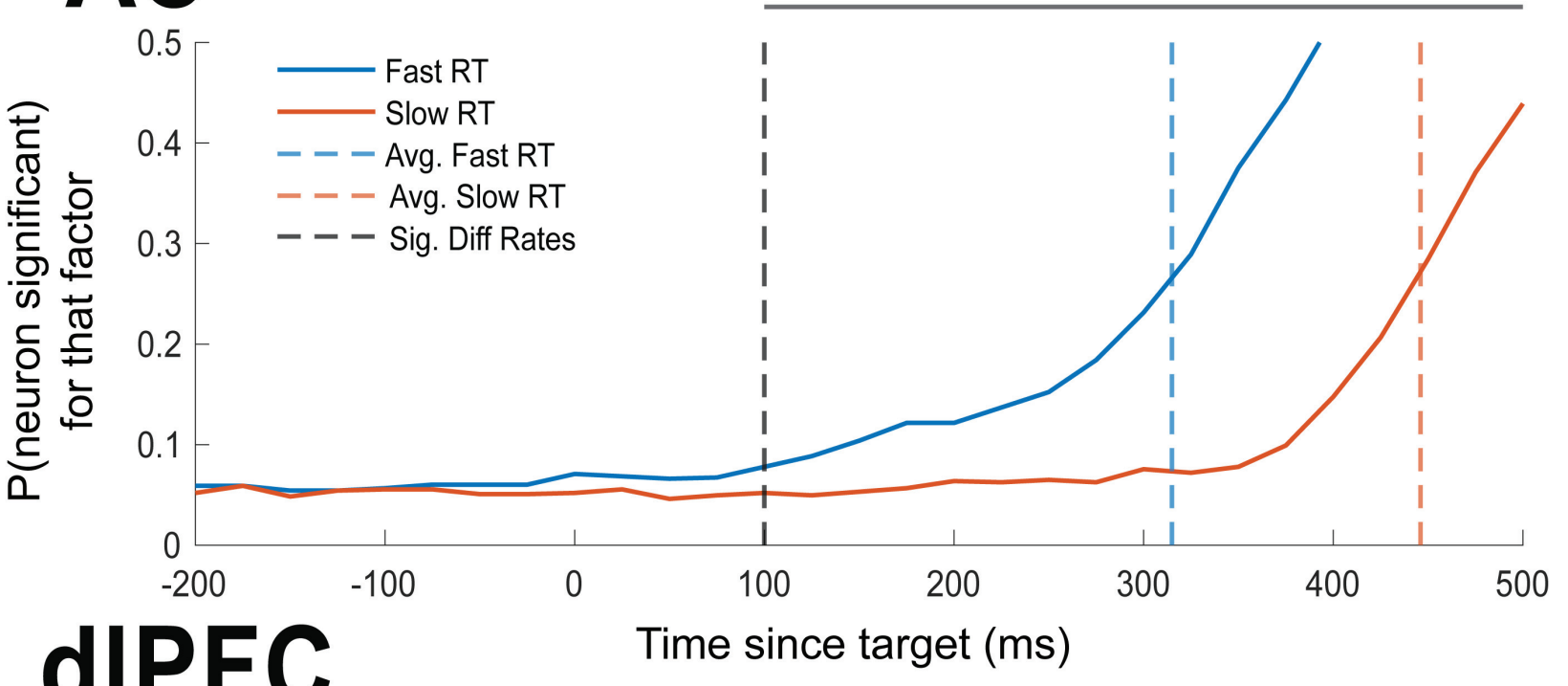

Time since target (ms)

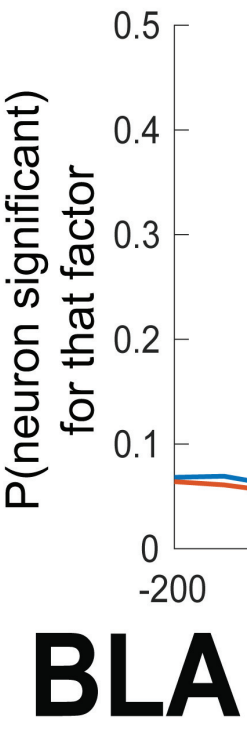

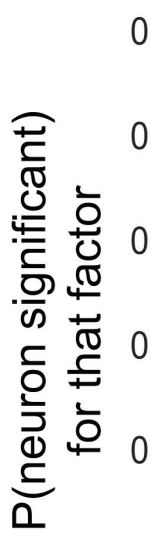
0.5

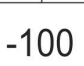

0
0
0
$\frac{1}{3}$
$\frac{1}{2}$

C

Time since target (ms)
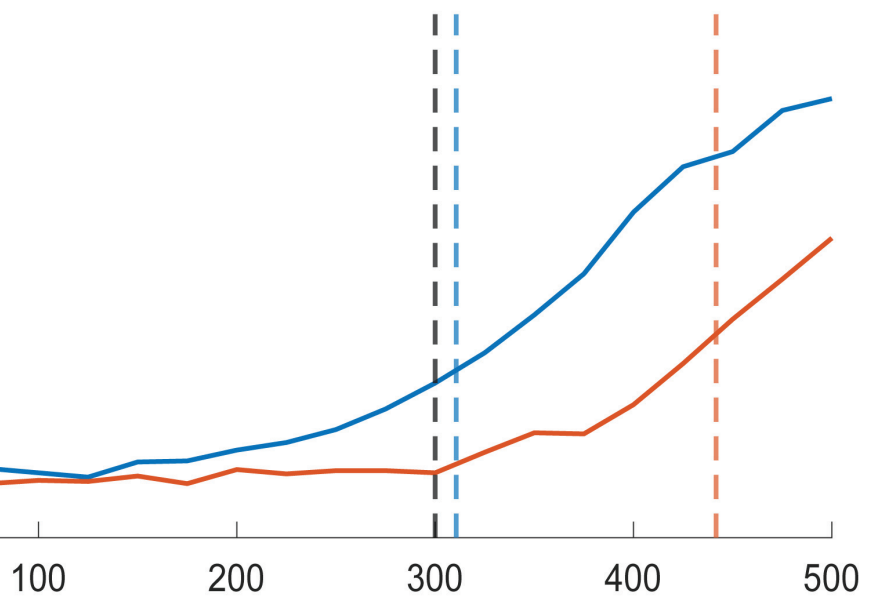

400

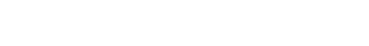


A

ᄃ AC

CD Cue selectivity Cue selectivity Target selectivity Response selectivity

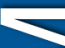

$\longrightarrow$

(O)

(a)

$+$

0

(1)

0

0

$<$

0

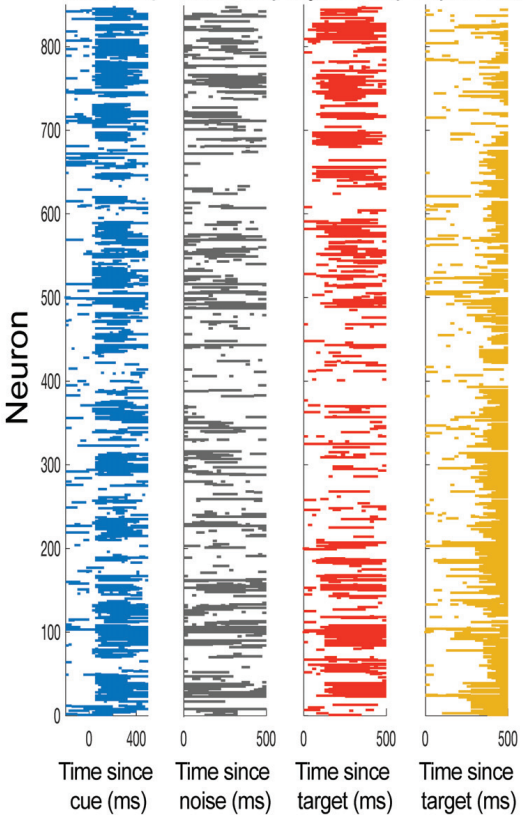

B

\section{dIPFC}

Cue selectivity Cue selectivity Target selectivity Response selectivity

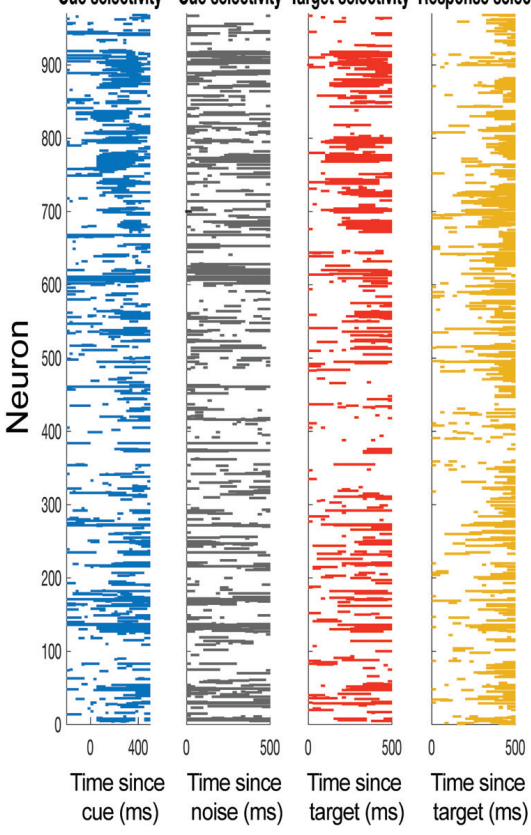

C

\section{BLA}

Cue selectivity Cue selectivity Target selectivity Response selectivity

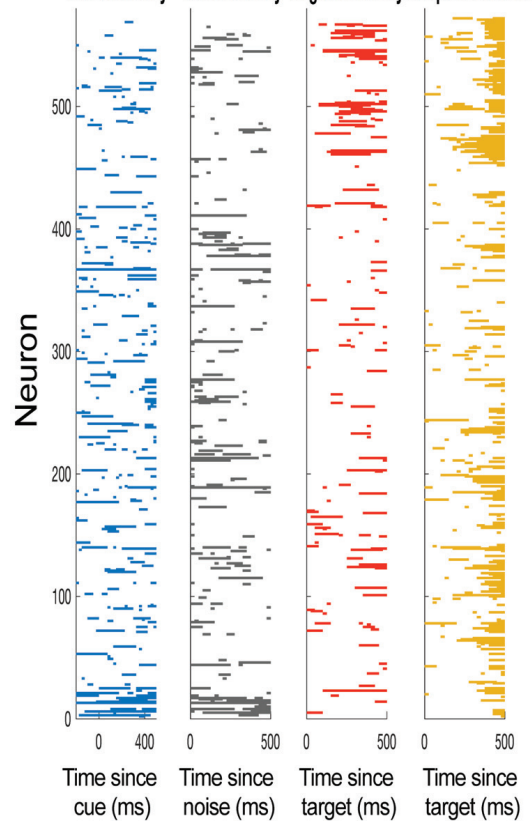




\section{Cue Epoch}

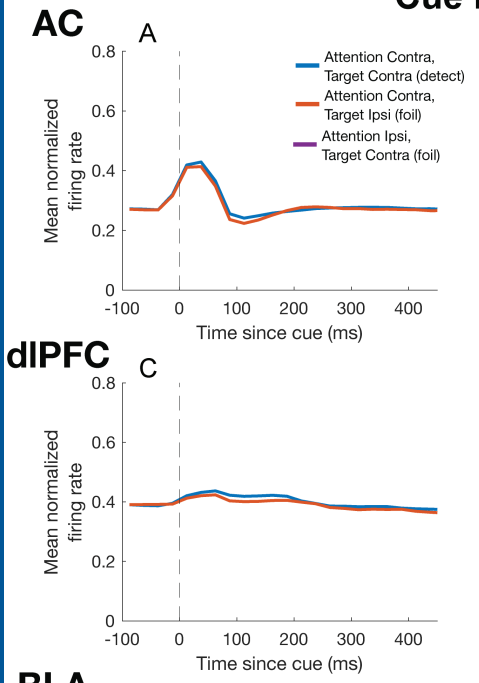

BLA $_{0.8}$ E

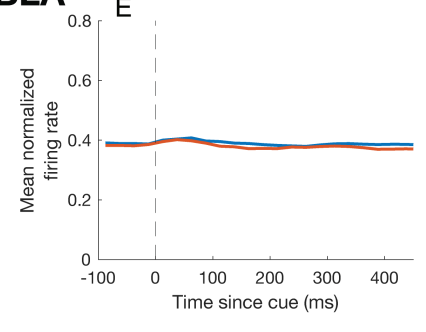

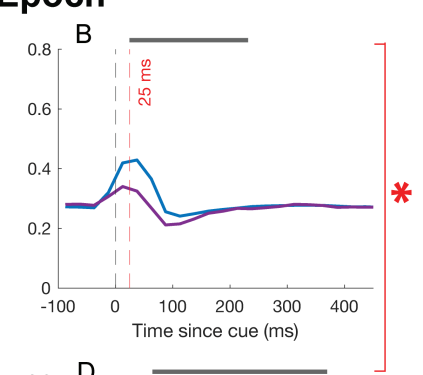
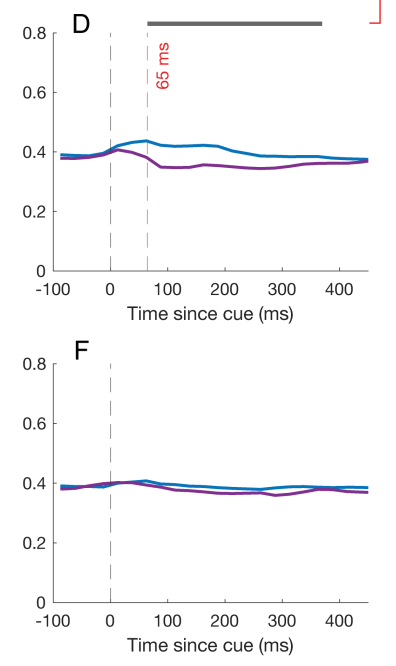

Target Epoch
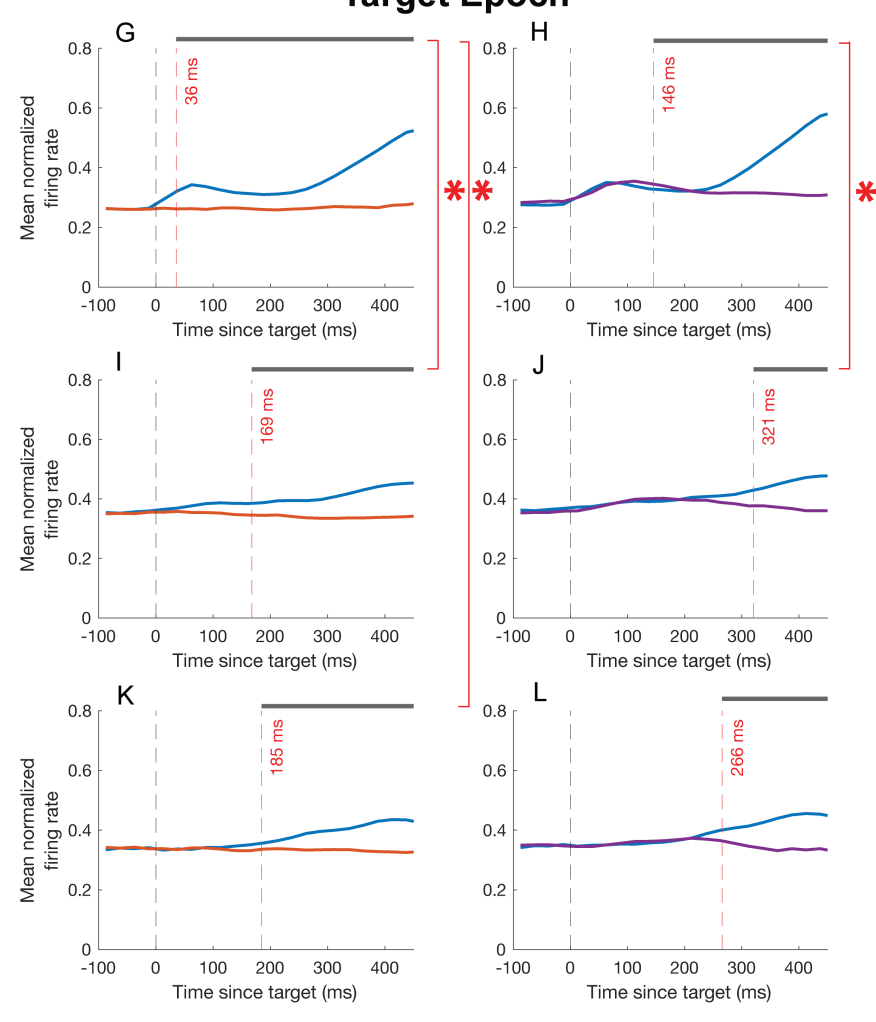
AC A B
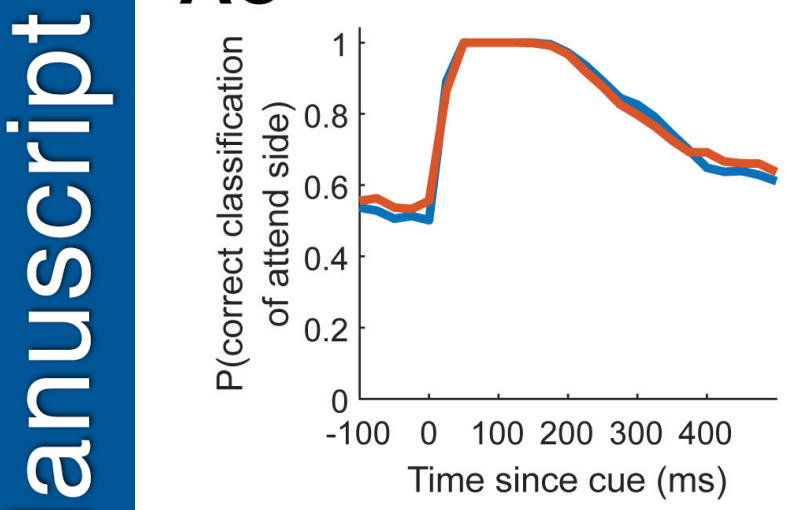

dIPFC D

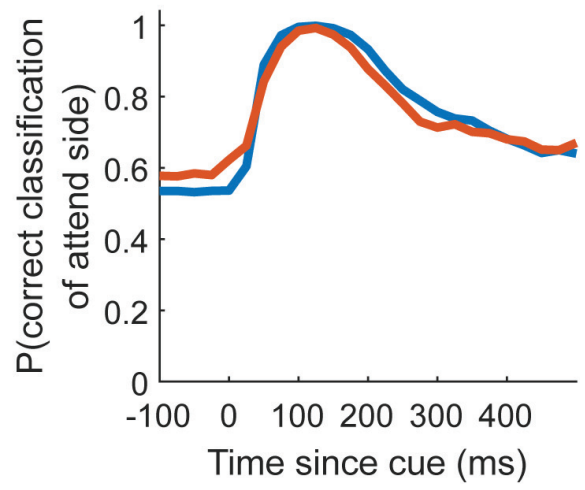

BLA

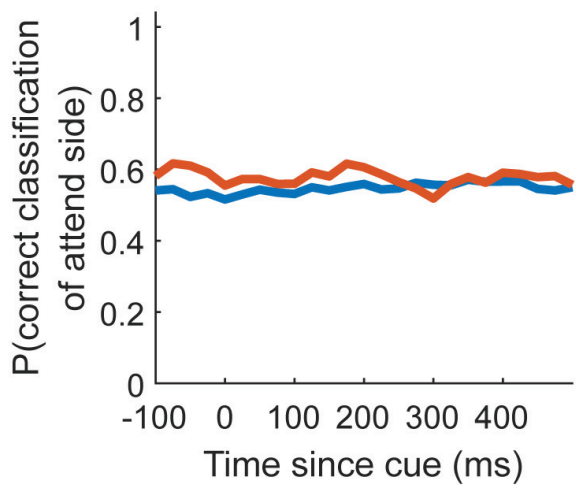

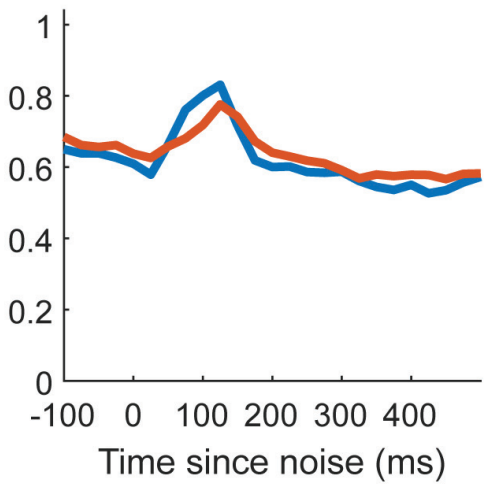

E

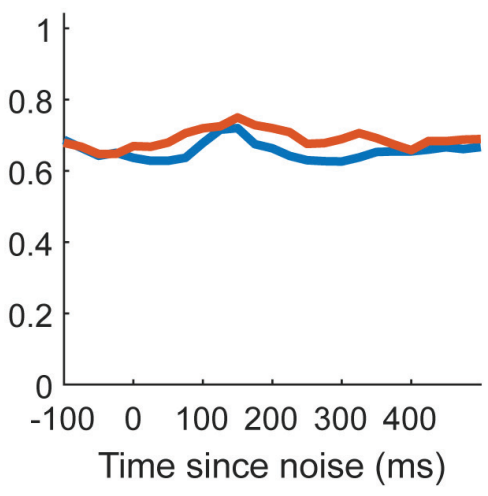

$\mathrm{H}$

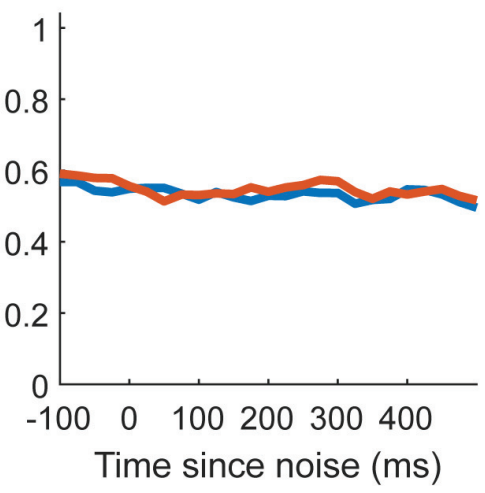

C

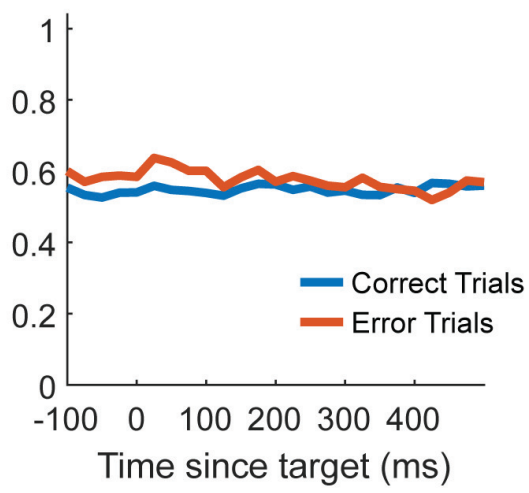

$F$

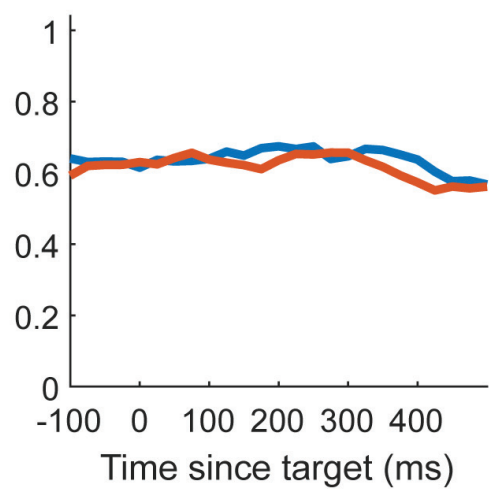

I

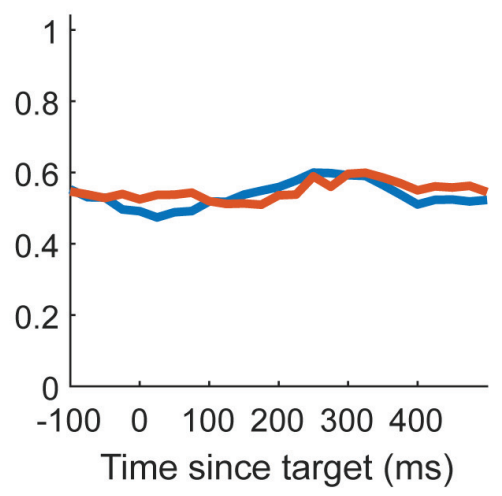


A

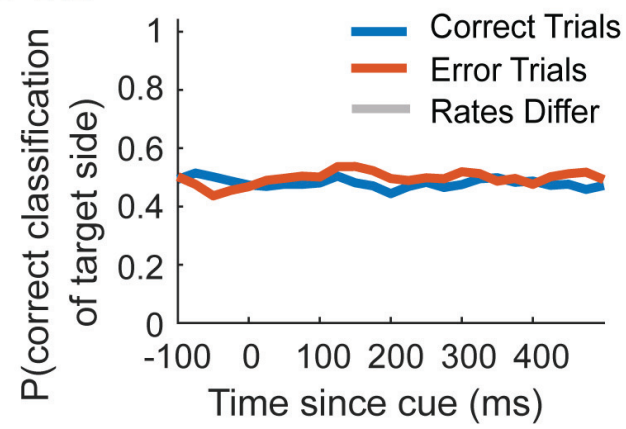

dIPFC D

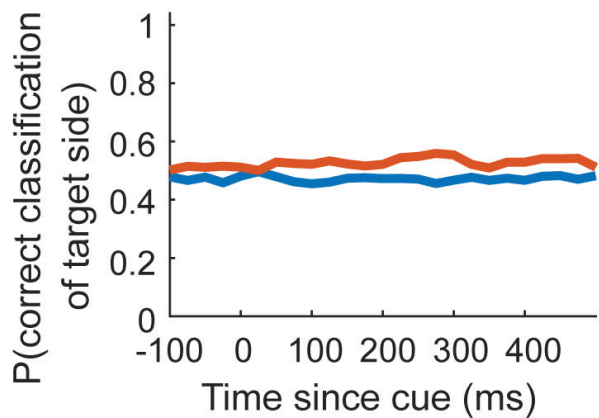

BLA

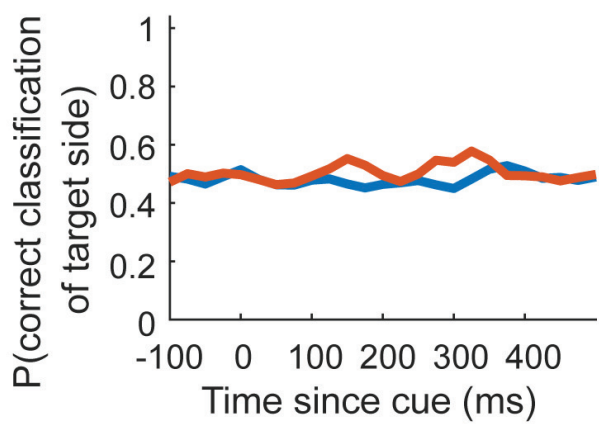

B

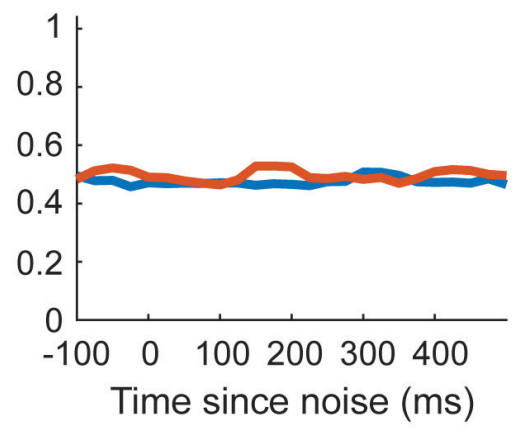

$E$

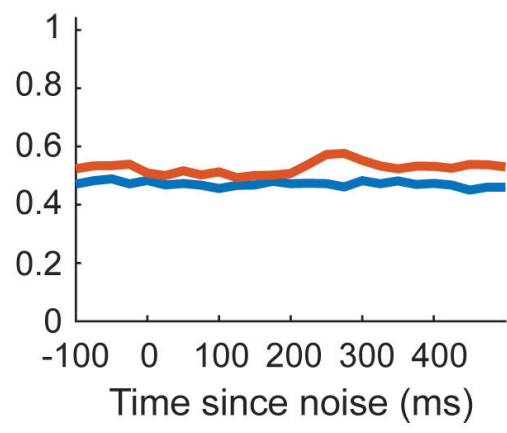

$\mathrm{H}$

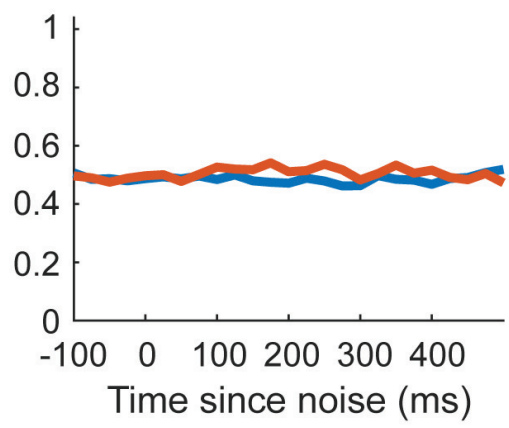

C

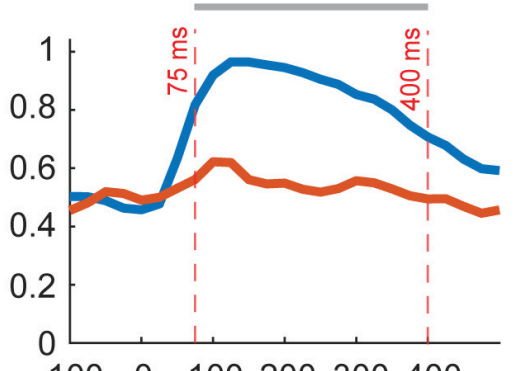

$-100 \quad 0 \quad 100200300400$

Time since target (ms)
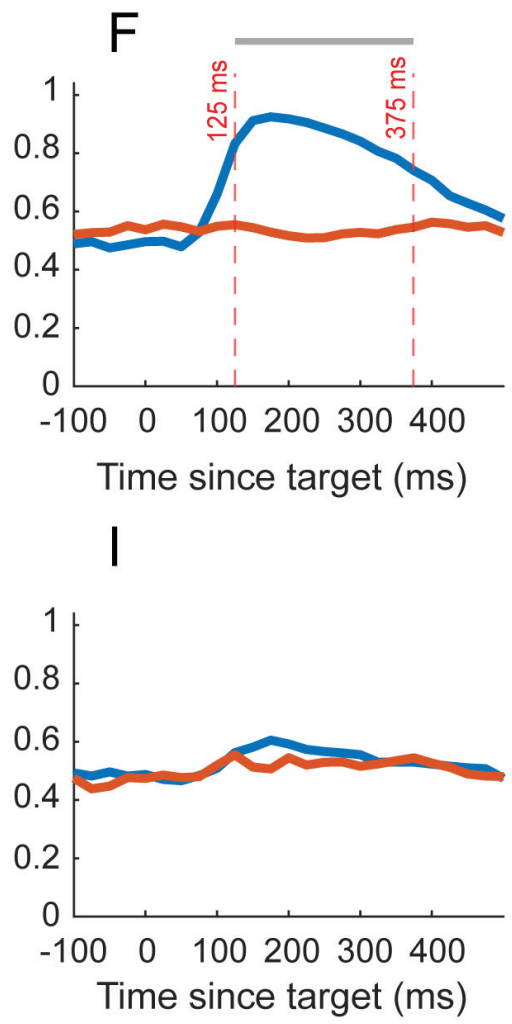
AC

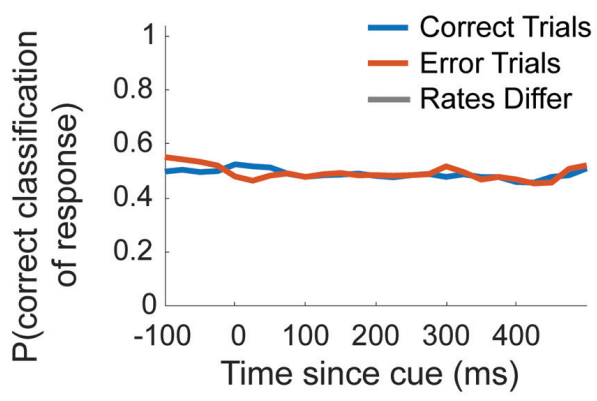

dIPFC D

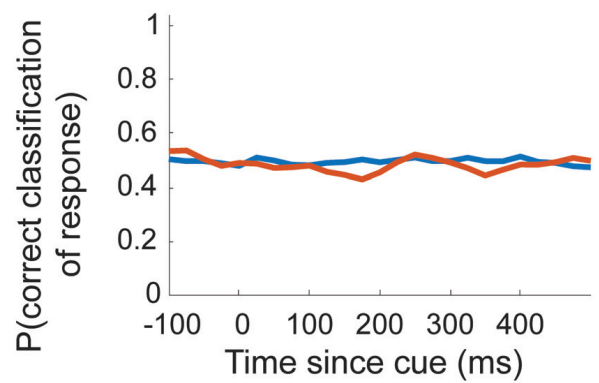

BLA G

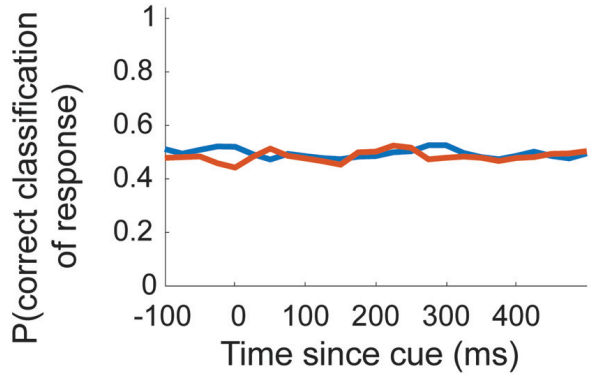

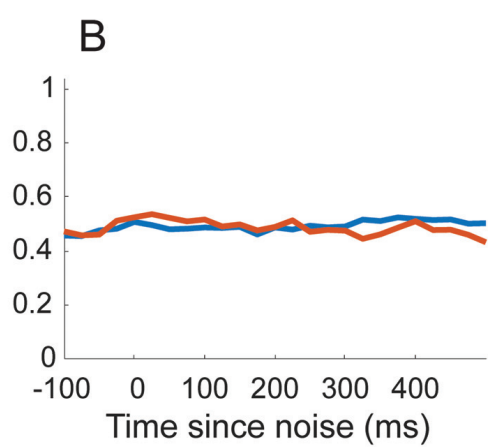

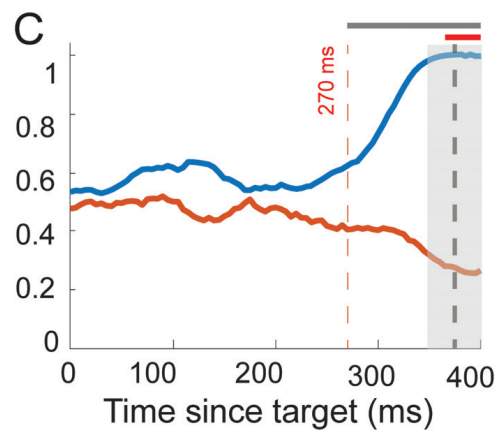

E
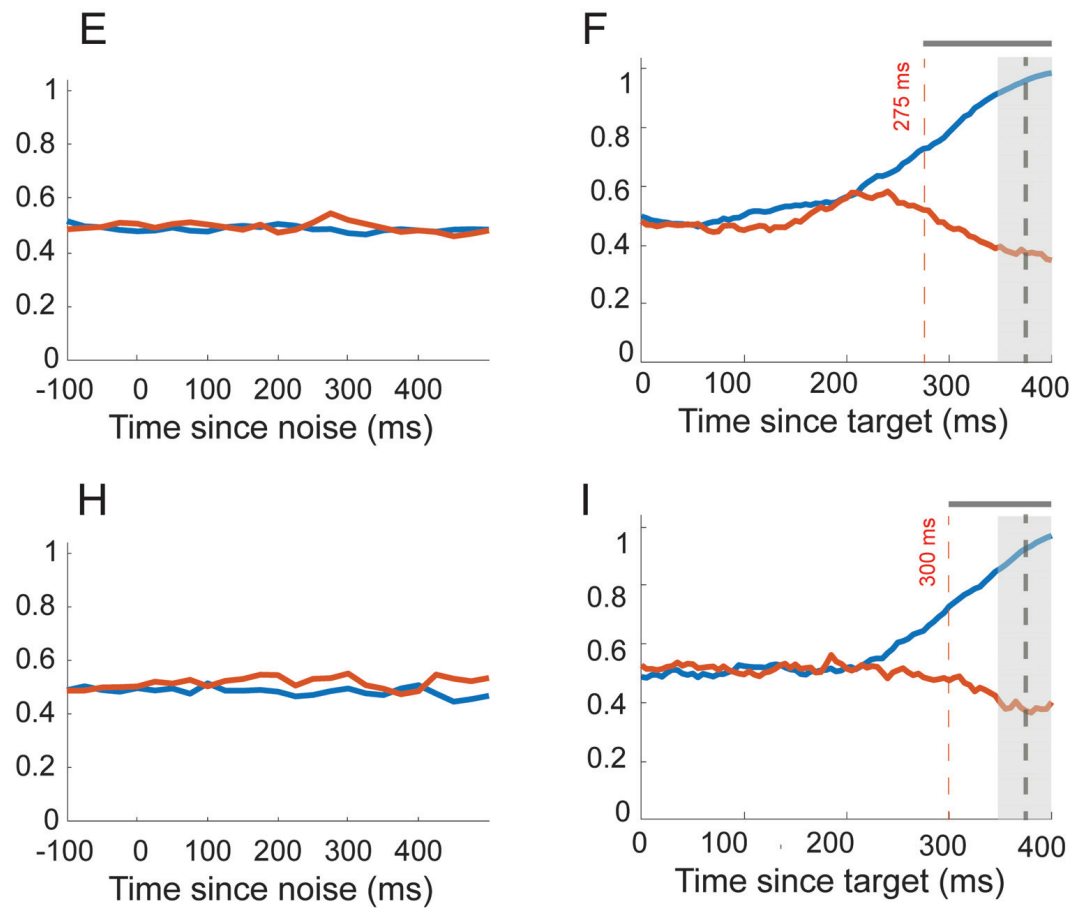
Cue L/R

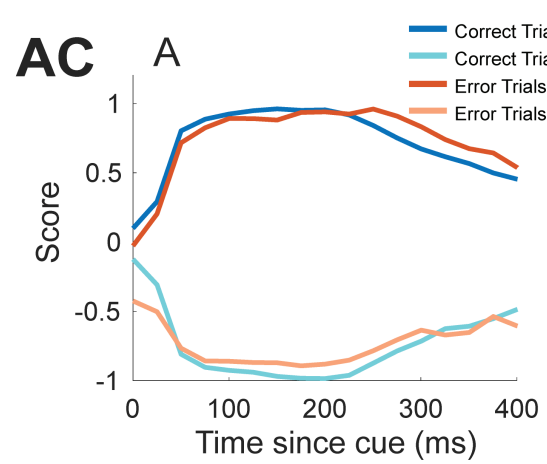

dIPFC $^{D}$

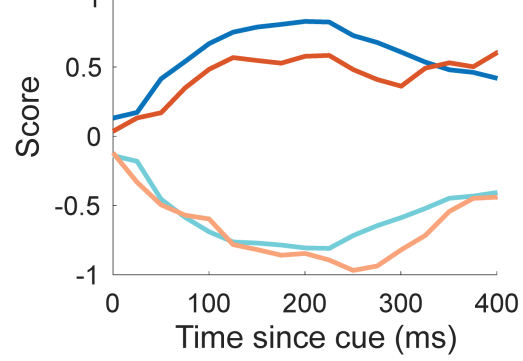

BLA $_{1}$

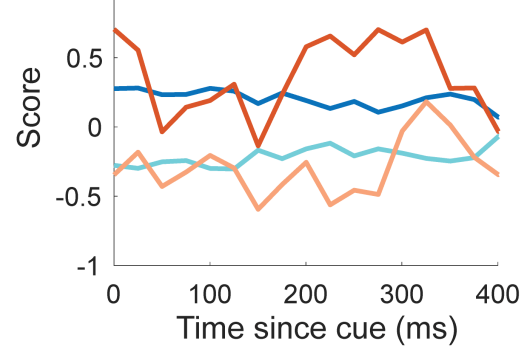

Target $L / R$

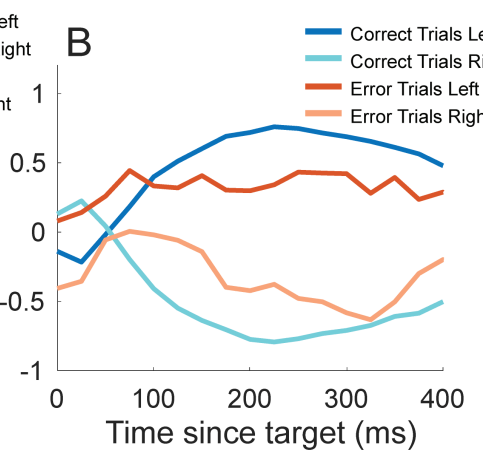

E
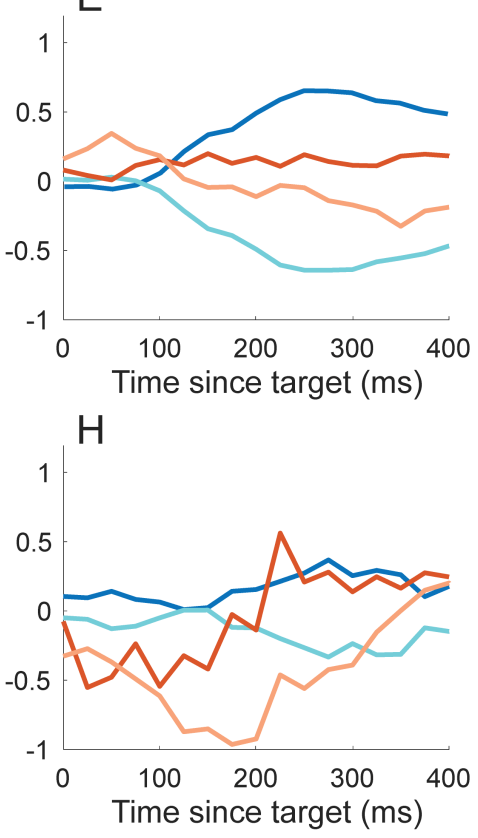

Response Y/N

C

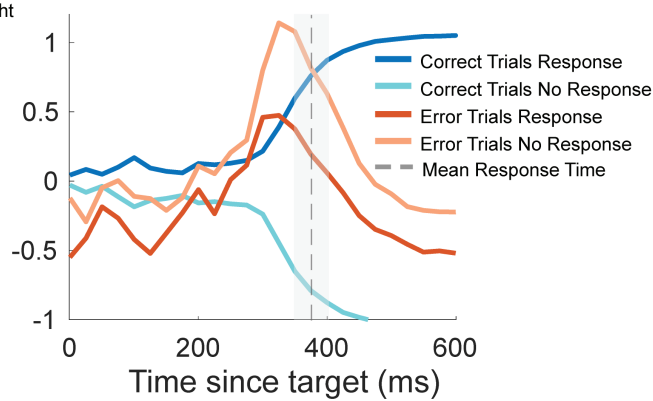

$\mathrm{F}$
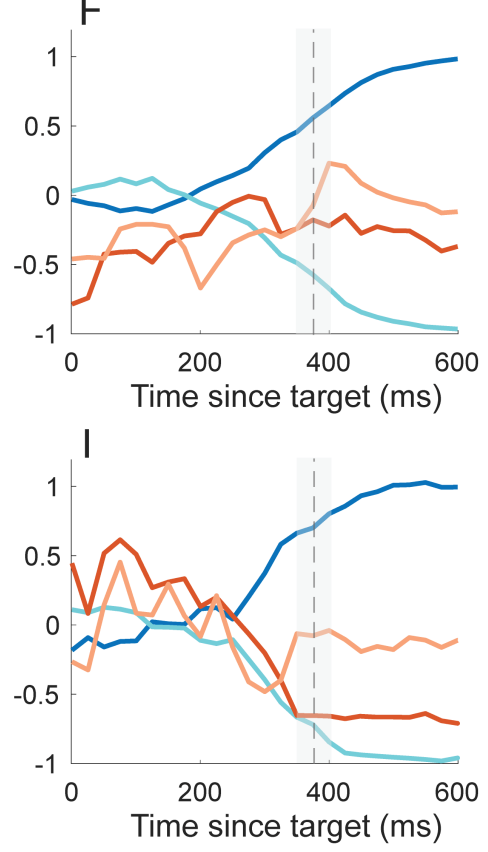

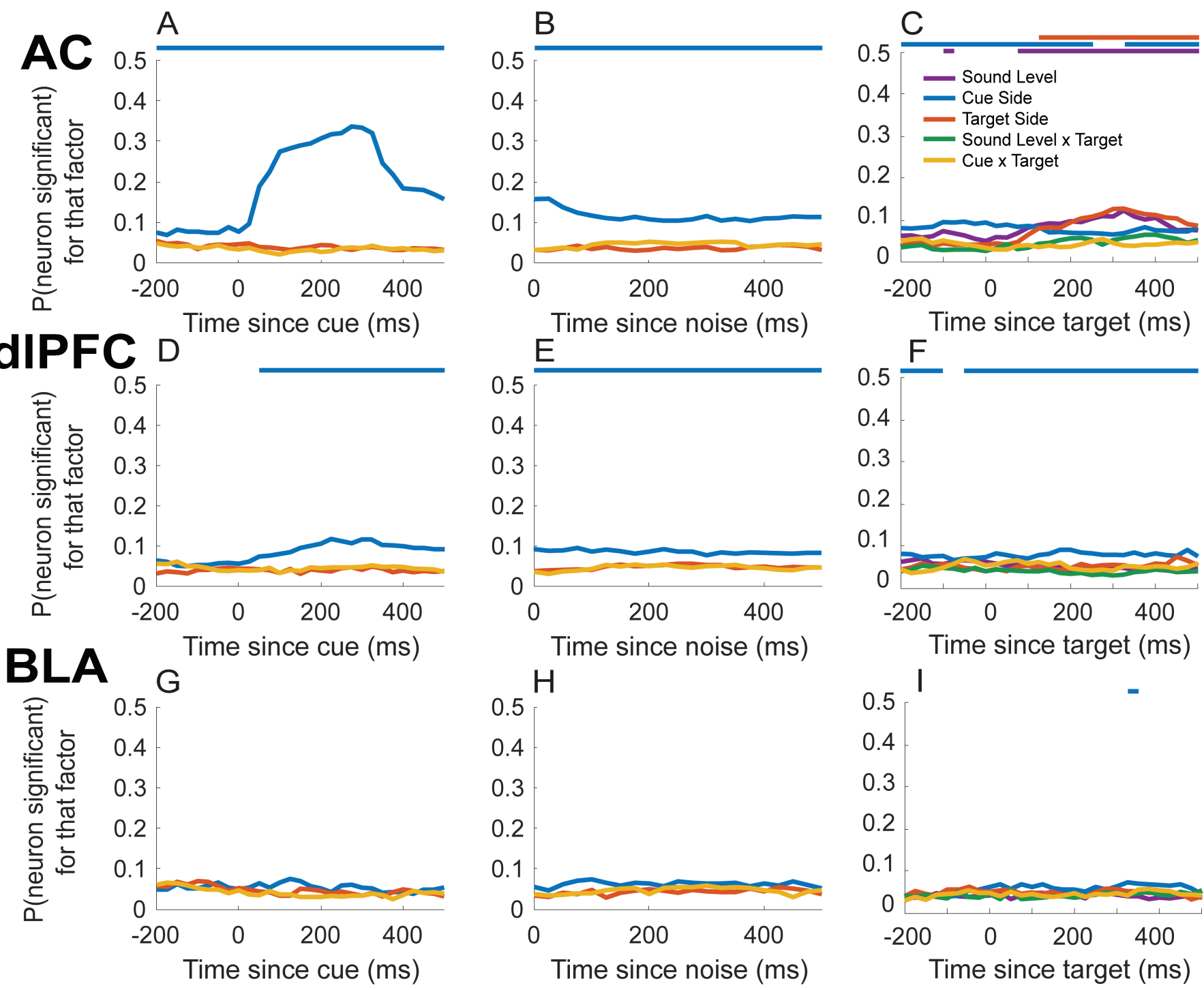


\section{Cue Epoch}

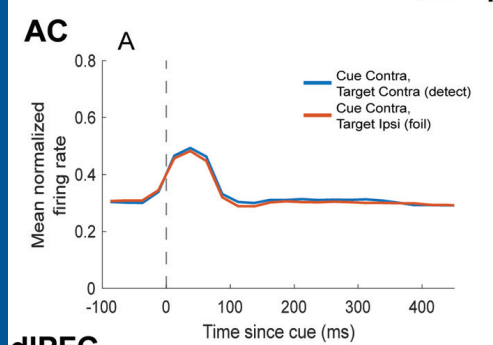

dIPFC
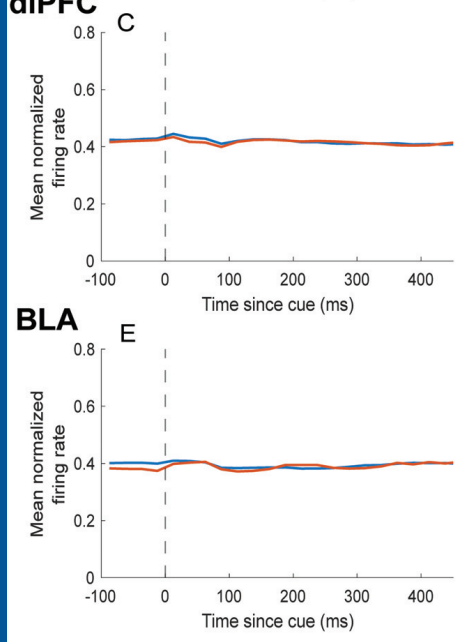
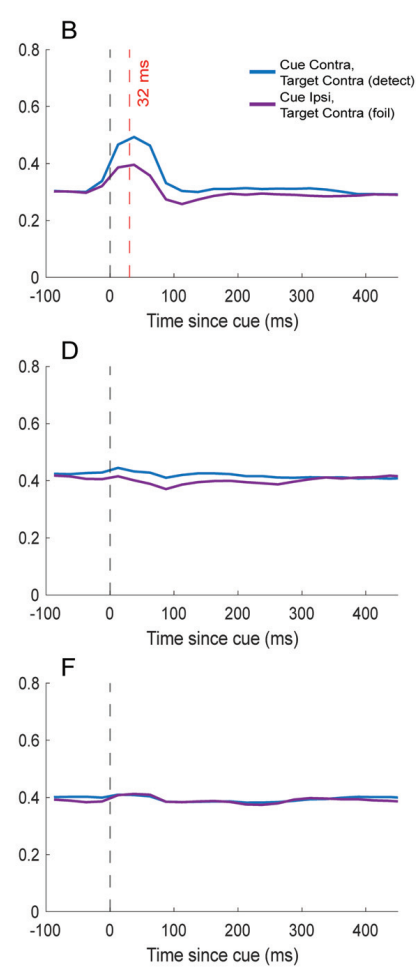

Target Epoch
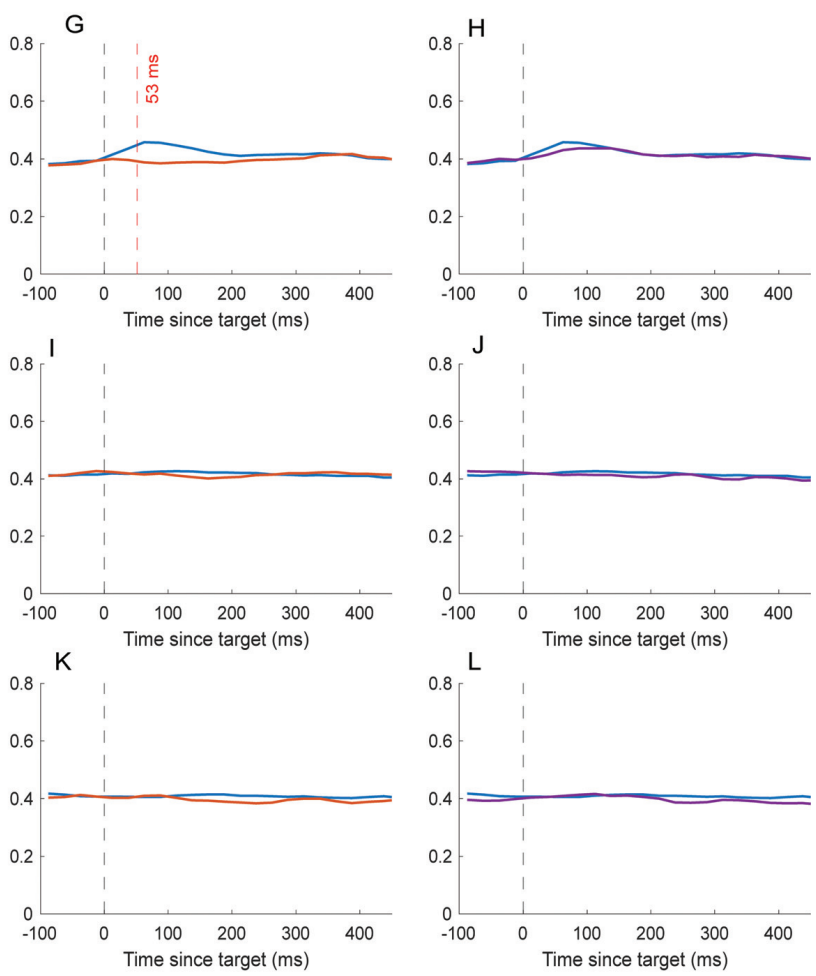


\begin{tabular}{lllllll} 
& \multicolumn{3}{c}{ Encoding cue } & \multicolumn{2}{c}{ Encoding delay } & Encoding target \\
\cline { 2 - 7 } also encoded & Delay & Target & Response & Target & Response & Response \\
\cline { 2 - 7 } AC & 26.70 & 17.05 & 5.40 & 16.9 & 8.81 & 5.83 \\
dIPFC & 30.90 & 10.11 & 4.49 & 9.80 & 3.92 & 5.80 \\
BLA & 25.00 & 8.33 & 2.08 & 5.77 & 7.69 & 8.00 \\
\hline
\end{tabular}

Note: all results displayed are in percentages

Table 1. Neuron selectivity to task variables by brain area. $2 \times 2$ ANOVA $(p<0.05)$ was performed using one bin per time period. A single time bin was chosen at the peak of the ANOVA curves (Fig. 3). Cue presentation included $150 \mathrm{~ms}-450 \mathrm{~ms}$ post-cue, the delay period included $100 \mathrm{~ms}-400 \mathrm{~ms}$ post-noise, target presentation included $50 \mathrm{~ms}-400 \mathrm{~ms}$ post-target presentation and choice period included $200-$ $500 \mathrm{~ms}$ post-target presentation. 